\title{
NON-SEPARABLE LATTICES, GABOR ORTHONORMAL BASES AND TILINGS
}

\author{
CHUN-KIT LAI AND AZITA MAYELI
}

\begin{abstract}
Let $K \subset \mathbb{R}^{d}$ be a bounded set with positive Lebesgue measure. Let $\Lambda=M\left(\mathbb{Z}^{2 d}\right)$ be a lattice in $\mathbb{R}^{2 d}$ with density dens $(\Lambda)=1$. It is well-known that if $M$ is a diagonal block matrix with diagonal matrices $A$ and $B$, then $\mathcal{G}\left(|K|^{-1 / 2} \chi_{K}, \Lambda\right)$ is an orthonormal basis for $L^{2}\left(\mathbb{R}^{d}\right)$ if and only if $K$ tiles both by $A\left(\mathbb{Z}^{d}\right)$ and $B^{-t}\left(\mathbb{Z}^{d}\right)$. However, there has not been any intensive study when $M$ is not a diagonal matrix. We investigate this problem for a large class of important cases of $M$. In particular, if $M$ is any lower block triangular matrix with diagonal matrices $A$ and $B$, we prove that if $\mathcal{G}\left(|K|^{-1 / 2} \chi_{K}, \Lambda\right)$ is an orthonormal basis, then $K$ can be written as a finite union of fundamental domains of $A\left(\mathbb{Z}^{d}\right)$ and at the same time, as a finite union of fundamental domains of $B^{-t}\left(\mathbb{Z}^{d}\right)$. If $A^{t} B$ is an integer matrix, then there is only one common fundamental domain, which means $K$ tiles by a lattice and is spectral. However, surprisingly, we will also illustrate by an example that a union of more than one fundamental domain is also possible. We also provide a constructive way for forming a Gabor window function for a given upper triangular lattice. Our study is related to a Fuglede's type problem in Gabor setting and we give a partial answer to this problem in the case of lattices.
\end{abstract}

Keywords: Non-separable lattices, Gabor orthonormal bases, tiling and spectral sets.

\section{INTRODUCTION}

Let $g \in L^{2}\left(\mathbb{R}^{d}\right)$, and let $\Lambda \subset \mathbb{R}^{2 d}$ be a countable subset. We define the Gabor system (also known as Weyl-Heisenberg system) $\mathcal{G}(g, \Lambda)$ with respect to $g$ and $\Lambda$ to be the collection of functions $\pi(a, b) g$ defined by combined time and frequency shifts of $g$ :

$$
\pi(a, b) g(x)=M_{b} T_{a} g=e^{2 \pi i\langle b, x\rangle} g(x-a) \quad(a, b) \in \Lambda .
$$

$\Lambda$ is also known as time-frequency set and the frequency shift is also called modulation. We say $g$ is an orthonormal Gabor window function with respect to $\Lambda$, or simply a window function, if $\mathcal{G}(g, \Lambda)$ is an orthonormal basis for $L^{2}\left(\mathbb{R}^{d}\right)$. See e.g. [3] or [13].

We call $\Lambda$ separable if it is of the form of $\Lambda=\mathcal{T} \times \Gamma$ for some countable subsets $\mathcal{T}$ and $\Gamma$ in $\mathbb{R}^{d}$. Gabor systems have been introduced for the first time in 1946 by Gabor [11] and are now fundamental objects in applied and computational harmonic analysis. Moreover, for $\mathcal{G}(g, \Lambda)$ to be a Gabor orthonormal basis, the (Beurling) density of $\Lambda$, denoted by dens $(\Lambda)$, must be 1 $[26]$.

The existence of a window function for a given lattice has been investigated for several special cases of $M$. The question of existence has been completely answered by Han and Wang [15] for separable lattices of the form $\Lambda=\mathcal{J} \times \mathcal{T}$ with $\operatorname{dens}(\Lambda)=1$. They answered the question by showing the existence of a common fundamental domain for two different lattices. Later, the

Date: November 20, 2018.

The work of the second author was partially supported by the PSC-CUNY Grant B cycles 47 and 48 . 
same authors partially answered the question for non-separable lattices (i.e. the lattices of not of the form of $\mathcal{J} \times \mathcal{T}$ ) for special cases of matrix $M$ [16]. Indeed, they proved that, when for example $M$ is a block triangular matrix, a window function $g$ exists and it can be chosen so that $|g|$ or $|\hat{g}|$ is the scalar multiple of a characteristic function. They also showed the existence of a window function with compact support for rational matrices $M$.

Given a subset $K \subset \mathbb{R}^{d}$, we denote by $\chi_{K}$ the indicator function of $K$ and by $|K|$ its Lebesgue measure. The main focus of this paper is the following. Suppose that $\Lambda=M\left(\mathbb{Z}^{2 d}\right) \subset \mathbb{R}^{2 d}$ is a full lattice with Beurling density $\operatorname{dens}(\Lambda)=|\operatorname{det}(M)|^{-1}=1$ and suppose that $\mathcal{G}\left(|K|^{-1 / 2} \chi_{K}, \Lambda\right)$ forms a Gabor orthonormal basis for $L^{2}\left(\mathbb{R}^{d}\right)$. What can we say about the structure of $K$ ? This question is related to the study of spectral sets and translational tiles which we will call the Fuglede-Gabor Problem later on.

Definition 1.1 (Spectral and tiling sets). A Lebesgue measurable set $K \subset \mathbb{R}^{d}$ with positive and finite measure is a spectral set in $\mathbb{R}^{d}$ if there is a countable set $B \subset \mathbb{R}^{d}$ (not necessarily unique) such that exponentials $\left\{e_{b}(x):=e^{2 \pi i\langle b, x\rangle}: b \in B, x \in K\right\}$ constitute an orthogonal basis for $L^{2}(K)$, i.e., the exponentials are mutual orthogonal and complete in $L^{2}(K)$. In this case $B$ is called a spectrum for $K$.

We say $K$ multi-tiles $\mathbb{R}^{d}$ by its translations if there is a countable set $\mathcal{J} \subset \mathbb{R}^{d}$ and integer $N \geq 1$ such that

$$
\sum_{t \in \mathcal{J}} \chi_{K}(x+t)=N \quad \text { a.e. } x \in \mathbb{R}^{d}
$$

If $N=1$, then $K$ tiles $\mathbb{R}^{d}$ and the set $\mathcal{J}$ is called tiling set for $K$ (For more details about multi-tiles, see e.g. [21]).

Spectral sets have been studied extensively in the recent years and their study has been reduced to the study of tiling sets by the Fuglede Conjecture or Spectral Set Conjecture [9] which asserts: $A$ set $K \subset \mathbb{R}^{d}$ with positive and finite measure is a spectral set if and only if $K$ tiles $\mathbb{R}^{d}$ by translations. Fuglede proved the conjecture in his celebrated 1974 paper [9] for the case when $K$ tiles by a lattice or $K$ has a spectrum which is a lattice. The Fuglede Conjecture led to considerable activity in the past three decades. In 2004, Tao [28] disproved the Fuglede conjecture for dimension 5 and higher, followed by Kolountzakis and Matolcsi's result [6, 22] where they proved that the conjecture fails in dimensions 3 and higher. For more recent results and historical comments see e.g. [1, 19].

Spectral sets and tiles appear naturally in the Gabor setting. Indeed, let $\Lambda=\mathcal{J} \times \mathcal{T}$ be a separable countable set (not necessarily a lattice) with $\operatorname{dens}(\Lambda)=1$ and let $\Omega$ be a compact set in $\mathbb{R}^{d}$ which tiles by $\mathcal{J}$ and is spectral for $\mathcal{T}$. For example, take $\Omega=[0,1]^{2}$ and $\mathcal{J}=\mathcal{T}=\mathbb{Z}^{2}$. Let $g$ be a function supported in $\Omega$. Then an easy calculation shows that the Gabor system $\mathcal{G}(g, \Lambda)$ is an orthonormal basis if $|g(x)|=|\Omega|^{-1 / 2} \chi_{\Omega}(x)$ (see also [30], Lemma 3.1). We call such Gabor bases standard. Liu and Wang [30] conjectured the converse of this result that for a compactly supported function $g$ and a countable separable set $\Lambda=\mathcal{J} \times \mathcal{T}$, if $\mathcal{G}(g, \Lambda)$ is an orthonormal basis for $L^{2}\left(\mathbb{R}^{d}\right)$, then there is a compact set $\Omega \subset \mathbb{R}^{d}$ such that $|g|$ is a constant multiple of $\chi_{\Omega}, \Omega$ tiles by $\mathcal{J}$ and is a spectral set for $\mathcal{T}$. Liu and Wang proved their conjecture when the support of $g$ is an interval. Dutkay and the first listed author recently proved that 
the Liu and Wang's conjecture is affirmative if $g$ is non-negative [5, Theorem 1.8]. However, the conjecture is still unsolved for general compactly supported $g$.

The following problem links the study of window functions associated with Gabor orthonormal bases to the tiling and spectral properties of sets.

Problem 1.2. (Fuglede-Gabor Problem) Let $K \subset \mathbb{R}^{d}$ be a measurable subset with positive and finite measure, and let $\Lambda \subset \mathbb{R}^{2 d}$ be a countable subset. If the Gabor family $\mathcal{G}\left(|K|^{-1 / 2} \chi_{K}, \Lambda\right)$ is an orthonormal basis for $L^{2}\left(\mathbb{R}^{d}\right)$, then $K$ tiles and is a spectral set.

Since the indicator function of a set is non-negative, Problem 1.2 is already affirmative if the time-frequency set is a separable countable sets using the results of Dutkay and the first listed author. Therefore we only focus on the case when the time-frequency set is non-separable. Moreover, although the problem does not require any boundedness assumption of $K$, our interest will mainly be focused on the set $K$ being bounded.

It is hard to speculate whether Problem 1.2 is true or not in its full generality. But from the point of view of Fuglede's result for lattices, we still hope that the Fuglede-Gabor problem is true for non-separable lattices as well. Unfortunately, after our intensive study, we found out that, similar to many notoriously difficult problems in Gabor analysis (see e.g. [14]), the FugledeGabor problem for lattices appears to be uneasy. This paper gives a partial answer towards the full solution together with some unexpected examples, as we explain below.

Main Results of the paper. Our main results will mostly be focused on the lower triangular block matrices since most matrices can be reduced to the lower triangular form:

$$
\Lambda=\left(\begin{array}{cc}
A & O \\
C & B
\end{array}\right)\left(\mathbb{Z}^{2 d}\right) \text {, and }|\operatorname{det}(A B)|=1
$$

(i.e. dens $(\Lambda)=1$ ). We will use $B^{-t}$ to denote the inverse transpose of the matrix $B$. Our first general key lemma is as follows, it will serve as a key step for our further analysis.

Lemma 1.3 (Key Lemma). Let $\Lambda=M\left(\mathbb{Z}^{2 d}\right)$ with $M$ an $2 d \times 2 d$ invertible lower triangular block matrix of the form (1.2). Suppose that $\mathcal{G}\left(|K|^{-1 / 2} \chi_{K}, \Lambda\right)$ is a Gabor orthonormal basis. Then there exists an integer $N \geq 1$ such that

$$
K=\bigcup_{j=1}^{N} D_{j}=\bigcup_{j=1}^{N} E_{j}
$$

where $D_{j}$ 's are fundamental domains of $B^{-t}\left(\mathbb{Z}^{d}\right)$ and $E_{j}$ 's are almost disjoint fundamental domains of $A\left(\mathbb{Z}^{d}\right)$ with $\left|D_{i} \cap D_{j}\right|=0$ and $\left|E_{i} \cap E_{j}\right|=0$ for all $i \neq j$. (i.e. $K$ multi-tiles $\mathbb{R}^{d}$ simultaneously by $A\left(\mathbb{Z}^{d}\right)$ and $B^{-t}\left(\mathbb{Z}^{d}\right)$.)

If we can prove that $N=1$, then $K$ will be a common fundamental domain for $A\left(\mathbb{Z}^{d}\right)$ and $B^{-t}\left(\mathbb{Z}^{d}\right)$ and this will imply that the Fuglede-Gabor problem holds. In particular, this is true when $A^{t} B$ is an integer matrix and $K$ is a bounded set, as our next result confirms.

Theorem 1.4. Let $K$ be a bounded measurable subset of $\mathbb{R}^{d}$ with positive measure, and let $\Lambda \subset$ $\mathbb{R}^{2 d}$ be a lower triangular lattice in (1.2). Suppose that $\mathcal{G}\left(|K|^{-1 / 2} \chi_{K}, \Lambda\right)$ is a Gabor orthonormal basis for $L^{2}\left(\mathbb{R}^{d}\right)$ and $A^{t} B$ is an integer matrix. Then $K$ tiles and is spectral. More precisely, $K$ is a common fundamental domain for $A\left(\mathbb{Z}^{d}\right)$ and $B^{-t}\left(\mathbb{Z}^{d}\right), K$ tiles by $A\left(\mathbb{Z}^{d}\right)$ and is spectral with spectrum $B\left(\mathbb{Z}^{d}\right)$. 
As a consequence of Theorem 1.4, we resolve the Fuglede-Gabor Problem 1.2 in dimension one for rational matrices and $K$ is bounded.

Theorem 1.5. Suppose that $K \subset \mathbb{R}$ is a bounded set with positive Lebesgue measure. Suppose that $\Lambda$ is a rational lattice in $\mathbb{R}^{2}$ with dens $(\Lambda)=1$. If $\mathcal{G}\left(|K|^{-1 / 2} \chi_{K}, \Lambda\right)$ is a Gabor orthonormal basis for $L^{2}(\mathbb{R})$, then $K$ tiles and is spectral.

We also have the following result for upper triangular block matrices using Theorem 1.4.

Theorem 1.6. Suppose that $K \subset \mathbb{R}^{d}$ is a bounded set with positive Lebesgue measure. Supposes that $\Lambda \subset \mathbb{R}^{2 d}$ is a lattice such that $\Lambda=\left(\begin{array}{cc}A & D \\ O & B\end{array}\right)\left(\mathbb{Z}^{2 d}\right)$ with dens $(\Lambda)=1, A^{-1} D$ symmetric rational matrix and $A^{t} B=I$. If $\mathcal{G}\left(|K|^{-1 / 2} \chi_{K}, \Lambda\right)$ is a Gabor orthonormal basis for $L^{2}\left(\mathbb{R}^{d}\right)$, then $K$ tiles and is spectral.

Theorem 1.4 may also be consider as a converse of [16, Lemma 4.1], which states that if $K$ is a common fundamental domain for the lattice $A\left(\mathbb{Z}^{d}\right)$ and $B^{-t}\left(\mathbb{Z}^{d}\right)$, then for any matrix $C$, the system $\mathcal{G}\left(|K|^{-1 / 2} \chi_{K}, \Lambda\right)$ is an Gabor orthonormal basis. Therefore one may naturally expect that $N=1$ in Theorem 1.3 is always the case. However, we will show that $N>1$ can actually happen with a suitable choice of $C$ if $A^{t} B$ is a rational matrix (see Example 6.1). This poses additional difficulty to solve the Fuglede-Gabor Problem for rational matrices in higher dimension, as we shall discuss it later. Finally, for a general matrix containing irrational entries, the answer to Fuglede-Gabor problem is completely open. We will discuss this in detail in Section 7.

Outline of the paper. We organize the paper as follows: After some definitions and recalling some known and basic facts about lattices and Gabor analysis in Section 2, in Section 3 we prove Theorem 1.3. The proof of Theorems 1.4 are 1.5 are presented in Section 4. In Section 5 we prove Theorem 1.6. Section 6 is devoted to examples illustrating the possibility for $N>1$. We conclude the paper with a series of open problems in Section 7 both for rational and irrational lattices as well as the full generality of the Fuglede-Gabor Problem. In our exposition, we discover that a new notion of completeness, which we will call exponential completeness, is crucial in studying the Fuglede-Gabor problem, we will give a short study in Appendix A. In Appendix B, we will show that the octagon will not produce any Gabor orthonormal basis using rational matrices.

\section{Preliminaries}

In this section, we will collect several basic definitions and results required for the rest of the paper. A full-rank lattice $\Lambda \subset \mathbb{R}^{d}$ is a discrete and countable subgroup of $\mathbb{R}^{d}$ with compact quotient group $\mathbb{R}^{d} / \Lambda$. A full-rank lattice in $\mathbb{R}^{d}$ is given by $\Lambda=M\left(\mathbb{Z}^{2 d}\right)$ for some $2 d \times 2 d$ invertible matrix $M \in G L(2 d, \mathbb{R})$. The density of $\Lambda$ is given by $\operatorname{dens}(\Lambda)=|\operatorname{det}(M)|^{-1}$.

Let $\Lambda$ be a lattice in $\mathbb{R}^{d}$. The dual lattice of $\Lambda$ is defined as

$$
\Lambda^{\perp}:=\left\{x \in \mathbb{R}^{2 d}:\langle\lambda, x\rangle \in \mathbb{Z}, \forall \lambda \in \Lambda\right\} .
$$

A direct calculation shows that $\Lambda^{\perp}=M^{-t}\left(\mathbb{Z}^{d}\right)$.

A fundamental domain of a lattice $\Lambda$ is a measurable set $\Omega$ in $\mathbb{R}^{d}$ which contains distinct representatives $(\bmod \Lambda)$ in $\mathbb{R}^{d}$, so that the any intersection of $\Omega$ with any coset $x+\Lambda$ has only 
one element. For the existence of a fundamental domain see [7, Theorem 1]. It is also evident that $\Omega$ tiles $\mathbb{R}^{d}$ with translations by $\Lambda$ and any other tiling set differs from $\Omega$ at most for a zero measure set.

1. A reduction lemma. For an invertible $d \times d$ matrix $A$, the operator $\mathcal{D}_{A}: L^{2}\left(\mathbb{R}^{d}\right) \rightarrow L^{2}\left(\mathbb{R}^{d}\right)$ defined by

$$
\mathcal{D}_{A} g(x):=|\operatorname{det}(A)|^{1 / 2} g(A x) .
$$

is unitary, i.e, $\mathcal{D}_{A}$ is onto and isometry $\left\|\mathcal{D}_{A} g\right\|=\|g\|$. The following lemma follows from a simple computation and is in general known. We will omit the detail of the proof.

Lemma 2.1. Let $\Lambda$ be a lattice such that

$$
\Lambda=\left(\begin{array}{cc}
A & D \\
C & B
\end{array}\right)\left(\mathbb{Z}^{2 d}\right)
$$

where $A$ is an invertible $d \times d$ matrix. Then

$$
\mathcal{G}(g, \Lambda)=\mathcal{D}_{A^{-1}} \mathcal{G}\left(\mathcal{D}_{A} g, \widetilde{\Lambda}\right)
$$

where

$$
\widetilde{\Lambda}=\left(\begin{array}{cc}
I & A^{-1} D \\
A^{t} C & A^{t} B
\end{array}\right)\left(\mathbb{Z}^{2 d}\right)
$$

Consequently, $\mathcal{G}(g, \Lambda)$ is a Gabor orthonormal basis if and only if $\mathcal{G}\left(\mathcal{D}_{A} g, \widetilde{\Lambda}\right)$ is a Gabor orthonormal basis.

In Lemma 2.1, if we let $D=O$ and $g(x)=|K|^{-1 / 2} \chi_{K}$, then the conclusion of the lemma shows that $\mathcal{G}\left(|K|^{-1 / 2} \chi_{K}, \Lambda\right)$ is an orthonormal basis for $L^{2}\left(\mathbb{R}^{d}\right)$ if and only if $\mathcal{G}\left(\left|A^{-1} K\right|^{-1 / 2} \chi_{A^{-1} K}, \widetilde{\Lambda}\right)$ is an orthonormal basis with

$$
\widetilde{\Lambda}=\left(\begin{array}{cc}
I & O \\
A^{t} C & A^{t} B
\end{array}\right)\left(\mathbb{Z}^{2 d}\right)
$$

We shall use this observation later.

2. Orthogonality implies completeness. The following proposition says that completeness automatically holds for a lattice of density one if we can establish the mutually orthogonality.

Proposition 2.2. Let $g \in L^{2}\left(\mathbb{R}^{d}\right)$, $\|g\|=1$ and $\Lambda \subset \mathbb{R}^{2 d}$ be a lattice with density dens $(\Lambda)=1$. Assume that $\mathcal{G}(g, \Lambda)$ is an orthonormal set. Then $\mathcal{G}(g, \Lambda)$ is complete.

For the proof of Proposition 2.2 we require the following lemma. Note that for a positive Borel measure $\mu$,

$$
f * \mu(x)=\int f(x-y) d \mu(y),
$$

given that the integral is well-defined. If $\mu=\sum_{\lambda \in \Lambda} \delta_{\lambda}$, then $\chi_{K} * \mu=1(\leq 1)$ if and only if $K$ tiles (packs) $\mathbb{R}^{d}$ by $\Lambda^{1}$. With this introduction we recall the following result.

\footnotetext{
${ }^{1}$ Recall that $K$ packs $\mathbb{R}^{d}$ by $\mathcal{J}$ if $\sum_{t \in \mathcal{J}} \chi_{K}(x-t) \leq 1$, a.e. $x \in \mathbb{R}^{d}$
} 
Lemma 2.3. ([24, Theorem 2.1]) Suppose that $f, g \in L^{1}\left(\mathbb{R}^{d}\right)$ are non-negative functions such that $\int f(x) d x=\int g(x) d x=1$. Suppose that for positive Borel measure $\mu$ on $\mathbb{R}^{d}$

$$
f * \mu \leq 1 \text { and } g * \mu \leq 1 .
$$

Then $f * \mu=1$ if and only if $g * \mu=1$.

Given $f, g \in L^{2}\left(\mathbb{R}^{d}\right)$, the short time Fourier transform is defined by

$$
V_{g} f(t, \xi)=\int f(x) \overline{g(x-t)} e^{-2 \pi i\langle\xi, x\rangle} d x, \quad(t, \xi) \in \mathbb{R}^{2 d}
$$

and it is a continuous function on $\mathbb{R}^{2 d}[13]$.

Proof of Proposition 2.2. The mutual orthogonality of $\mathcal{G}(g, \Lambda)$ implies the Bessel inequality of the system:

$$
\sum_{(t, \xi) \in \Lambda}\left|V_{g} f(t, \xi)\right|^{2} \leq\|f\|^{2}, \quad \forall f \in L^{2}\left(\mathbb{R}^{d}\right)
$$

Let $s, \xi \in \mathbb{R}^{d}$. The inequality (2.3) for $e^{2 \pi i\langle\nu, x\rangle} f(x-s)$ in the place of $f$ yields

$$
\sum_{(t, \xi) \in \Lambda}\left|V_{g} f(t-s, \xi-\nu)\right|^{2} \leq\|f\|^{2} \quad \forall(s, \nu) \in \mathbb{R}^{2 d} .
$$

Hence, $\left|V_{g} f\right|^{2} * \delta_{\Lambda} \leq\|f\|^{2}$. Take $G:=\|f\|^{-2}\left|V_{g} f\right|^{2}$. Then $\int_{\mathbb{R}^{2 d}} G(z) d z=1$ and $G * \delta_{\Lambda} \leq 1$. On the other hand, $\Lambda$ is a lattice with density 1 . Let $\Omega \subset \mathbb{R}^{2 d}$ be any fundamental domain for $\Lambda$. Then $|\Omega|=1$ and it tiles $\mathbb{R}^{2 d}$ by $\Lambda$. Therefore $\chi_{\Omega} * \delta_{\Lambda}=1$. Now Lemma 2.3 implies that $G * \delta_{\Lambda}=1$. But this is equivalent to the completeness of the system $\mathcal{G}(g, \Lambda)$ and we are done.

3. Some reduction to lower triangular block matrices. The following result is due to Han and Wang which states that any invertible integer matrix can be converted into a lower triangular integer matrix. We will need it in later sections. An integer matrix $P$ is called unimodular if $\operatorname{det} P=1$.

Lemma 2.4. ([16], Lemma 4.4) Let $M$ be an $d \times d$ invertible integer matrix. Then there is an $d \times d$ unimodular matrix $P$ such that $M P$ is a lower triangular integer matrix.

As a corollary of Lemma 2.4 we can show that any rational matrix can be represented as a lower triangular rational matrix.

Corollary 2.5. Let $M$ be an $d \times d$ invertible rational matrix. Then there is a lower triangular rational matrix $N$ such that $M\left(\mathbb{Z}^{d}\right)=N\left(\mathbb{Z}^{d}\right)$.

Henceforth, we shall say matrix $M$ is equivalent to $N$ if $M\left(\mathbb{Z}^{d}\right)=N\left(\mathbb{Z}^{d}\right)$.

4. Exponential Completeness. Recall that a collection of functions $\left\{\varphi_{n}\right\}$ is said to be complete in $L^{2}(\Omega)$ if $\left\langle f, \varphi_{n}\right\rangle=0$ for all $n$ implies that $f=0$ a.e. on $L^{2}(\Omega)$. Given $f \in L^{2}\left(\mathbb{R}^{d}\right)$, the Fourier transform of $f$ is defined to be $\widehat{f}(\xi)=\int_{\mathbb{R}^{d}} f(x) e^{-2 \pi i\langle\xi, x\rangle} d x$. In our study, we will need to following weaker notion of the completeness property. 
Definition 2.6. Let $\Lambda$ be a countable set and let $\Omega$ be a Lebesgue measurable set with positive finite measure. We say that the set of exponentials $\left\{e^{2 \pi i\langle\lambda, x\rangle}: \lambda \in \Lambda\right\}$ (or $\Lambda$ ) is exponentially complete for $L^{2}(\Omega)$ if there does not exist any $\xi \in \mathbb{R}^{d}$ such that

$$
\widehat{\chi_{\Omega}}(\lambda-\xi)=\int_{\Omega} e^{2 \pi i\langle\xi, x\rangle} e^{-2 \pi i\langle\lambda, x\rangle} d x=0, \forall \lambda \in \Lambda .
$$

Remark 2.7. Throughout the paper, we will see that exponential completeness plays an important role in constructing Gabor orthonormal basis using non-separable lattices. If a countable set of exponentials is complete for $L^{2}(\Omega)$, then it must be exponentially complete (otherwise $e^{2 \pi i\langle\xi, x\rangle}$ will be orthogonal to all $e^{2 \pi i\langle\lambda, x\rangle}$ contradicting completeness). However, the converse is not true. For example, the set of exponentials associated to the lattice $\Lambda=\sqrt{2} \mathbb{Z}$ is exponentially complete in $L^{2}([0,1])$, but it is not complete in it (see Lemma 4.2). In Appendix 1, we will give a short study about the exponential completeness for lattices in $L^{2}[0,1]^{d}$.

\section{Proof of Lemma 1.3 - Union of fundamental domains}

We now prove our Theorem 1.3. It follows from two theorems in two separate fields. The first one is taken from the study of Fuglede's problems. It was first proved by Jorgensen and Pedersen [20, Theorem 6.2 (b)] and then Lagarias and Wang [29, Theorem 2.1] gave a simpler proof.

Theorem 3.1. Let $\Omega \subset \mathbb{R}^{d}$ be a Lebesgue measurable set with positive finite measure. Suppose $\Gamma$ is a full-rank lattice such that $\Gamma \subseteq\left\{\xi: \widehat{\chi_{\Omega}}(\xi)=0\right\} \cup\{0\}$. Then $\Omega=\bigcup_{j=1}^{N} D_{j}$, up to measure zero, where $D_{j}$ are fundamental domains for the dual lattice $\Gamma^{\perp},\left|D_{i} \cap D_{j}\right|=0, i \neq j$ and $N=|\Omega| /\left|D_{j}\right|$.

Given a lattice $\Lambda=M\left(\mathbb{Z}^{2 d}\right)$, the adjoint lattice $\Lambda^{\circ}$ is a lattice such that

$$
J\left(\Lambda^{\circ}\right)=\Lambda^{\perp}
$$

where $J=\left(\begin{array}{cc}O & -I \\ I & O\end{array}\right)$. In other words, $\Lambda^{\circ}=J^{-1} M^{-t}\left(\mathbb{Z}^{d}\right)$.

The Ron-Shen duality theorem [27] is well-known in Gabor analysis. It was first proved over symplectic lattices, it is known to be true over any lattice (see e.g [14, Theorem 2.3] for a proof by Poisson Summation Formula). We will need the following version of duality theorem.

Theorem 3.2. $\mathcal{G}(g, \Lambda)$ is a Gabor orthonormal basis if and only if $\mathcal{G}\left(g, \Lambda^{\circ}\right)$ is a Gabor orthonormal basis.

Sketch of Proof. This statement is well-known. Here we provide a simple proof based on [14, Theorem 2.3] and Proposition 2.2. Since $\left(\Lambda^{\circ}\right)^{\circ}=\Lambda$, both sides of the statements are symmetric and we just need to prove one side of the equivalence. Suppose that $\mathcal{G}(g, \Lambda)$ is a Gabor orthonormal basis. Then $g$ is the only dual window with the property that

$$
\langle g, \pi(\mu)(g)\rangle=0 \forall \mu \in \Lambda^{\circ} \backslash\{0\}
$$

(by [14, Theorem 2.3]). This means that for all distinct $\mu, \mu^{\prime} \in \Lambda^{\circ},\left\langle\pi(\mu) g, \pi\left(\mu^{\prime}\right)(g)\right\rangle=c\langle g, \pi(\mu-$ $\left.\left.\mu^{\prime}\right)(g)\right\rangle=0$ ( $c$ is some unimodular constant). Thus $\mathcal{G}\left(g, \Lambda^{\circ}\right)$ is mutually orthogonal. As $\mathcal{G}(g, \Lambda)$ is a Gabor orthonormal basis, $\|g\|=1$ and $\operatorname{dens}\left(\Lambda^{\circ}\right)=\operatorname{dens}(\Lambda)=1$, by Proposition $2.2, \mathcal{G}\left(g, \Lambda^{\circ}\right)$ is complete and is thus an orthonormal basis. 
For a lower triangular lattice $\Lambda=\left(\begin{array}{cc}A & O \\ C & B\end{array}\right) \mathbb{Z}^{2 d}$, the adjoint lattice $\Lambda^{\circ}$ is also a lower triangular and it can be calculated as follows:

$$
\Lambda^{\circ}=\left(\begin{array}{cc}
O & I \\
-I & O
\end{array}\right)\left(\begin{array}{cc}
A^{-t} & -A^{-t} C^{t} B^{-t} \\
O & B^{-t}
\end{array}\right)\left(\mathbb{Z}^{2 d}\right)=\left(\begin{array}{cc}
O & B^{-t} \\
-A^{-t} & A^{-t} C^{t} B^{-t}
\end{array}\right)\left(\mathbb{Z}^{2 d}\right) .
$$

From the other hand we can write

$$
\left(\begin{array}{cc}
O & B^{-t} \\
-A^{-t} & A^{-t} C^{t} B^{-t}
\end{array}\right)=\left(\begin{array}{cc}
B^{-t} & O \\
A^{-t} C^{t} B^{-t} & A^{-t}
\end{array}\right)\left(\begin{array}{cc}
O & I \\
-I & O
\end{array}\right)\left(\mathbb{Z}^{2 d}\right)
$$

$\operatorname{But}\left(\begin{array}{cc}O & I \\ -I & O\end{array}\right)\left(\mathbb{Z}^{2 d}\right)=\mathbb{Z}^{2 d}$, therefore we have

$$
\Lambda^{\circ}=\left(\begin{array}{cc}
B^{-t} & O \\
A^{-t} C^{t} B^{-t} & A^{-t}
\end{array}\right)\left(\mathbb{Z}^{2 d}\right) .
$$

Proof of Theorem 1.3. The orthogonality of the Gabor system implies that

$$
\int_{K} e^{-2 \pi i\langle B n, x\rangle} d x=0 \quad \forall n \in \mathbb{Z}^{d} \backslash\{0\} .
$$

By Theorem 3.1, (3.1) implies that $K$ can be written as $K=\bigcup_{j=1}^{N} D_{j}$, where $D_{j}$ is a fundamental domain for $B^{-t}\left(\mathbb{Z}^{d}\right)$. On the other hand, by the duality Theorem $3.2, \mathcal{G}\left(|K|^{-1 / 2} \chi_{K}, \Lambda^{\circ}\right)$ is a Gabor orthonormal basis, too. Similarly, the exponentials $\left\{e^{2 \pi i\left\langle A^{-t} n, x\right\rangle}: n \in \mathbb{Z}^{d}\right\}$ are mutually orthogonal in $L^{2}(K)$. Hence, by Theorem 3.1, we have $K=\bigcup_{j=1}^{M} E_{j}$ where $E_{j}$ 's are fundamental domains for $A\left(\mathbb{Z}^{d}\right)$. Since $\operatorname{det}(A B)=1$ we conclude that $\left|D_{i}\right|=\left|E_{j}\right|, \forall i, j$. Since $|K|<\infty$, the latter forces that $M=N$, hence the proof of the theorem is completed.

\section{Proof of Theorems 1.4 And 1.5 - Lower triangular matrices}

To prove Theorem 1.4, first we shall apply some preliminary reductions to the theorem, as follows. Due to Lemma 2.1 and the hypothesis of the theorem on the matrices $A$ and $B$, for the proof it is sufficient to assume that $\Lambda=\left(\begin{array}{cc}I & O \\ C & B\end{array}\right)\left(\mathbb{Z}^{2 d}\right)$, where $B$ is an invertible matrix with integer entries (since originally $A^{t} B$ has integer entries by the assumption of Theorem 1.4). Notice by the density condition $\operatorname{dens}(\Lambda)=1$, we have $|\operatorname{det}(B)|=1$. Thus $B^{-1}$ is also an integral matrix with determinant 1 and we have $\mathbb{Z}^{2 d}=\left(\begin{array}{cc}I & O \\ O & B^{-1}\end{array}\right)\left(\mathbb{Z}^{2 d}\right)$. Thus we can rewrite $\Lambda$ as follows:

$$
\Lambda=\left(\begin{array}{cc}
I & O \\
C & B
\end{array}\right)\left(\begin{array}{cc}
I & O \\
O & B^{-1}
\end{array}\right)\left(\mathbb{Z}^{2 d}\right)=\left(\begin{array}{cc}
I & O \\
C & I
\end{array}\right)\left(\mathbb{Z}^{2 d}\right)
$$

Therefore to prove Theorem 1.4 it suffices to consider $\Lambda=\left(\begin{array}{cc}I & O \\ C & I\end{array}\right)\left(\mathbb{Z}^{2 d}\right)$. In this case if $\mathcal{G}\left(|K|^{-1 / 2} \chi_{K}, \Lambda\right)$ is an orthonormal basis, then by Theorem 1.3 we must have $K=\bigcup_{j=1}^{N} E_{j}$ where $E_{j}$ 's are disjoint fundamental domains of $\mathbb{Z}^{d}$. In particular, $K$ is a multi-tile for $\mathbb{R}^{d}$ with respect to $\mathbb{Z}^{d}$, i.e.,

$$
\sum_{k \in \mathbb{Z}^{d}} \chi_{K}(x+k)=N \text { a.e. } x \in[0,1)^{d} .
$$


Our goal is to show that $N=1$. For this, the following proposition will serve a key role.

Proposition 4.1. Suppose $K$ is a bounded set which multi-tiles $\mathbb{R}^{d}$ with respect to $\mathbb{Z}^{d}$ at level $N$, i.e. (4.1) holds. Suppose that $N>1$. Then there exists $m \in \mathbb{Z}^{d}$ such that

(1) $K \cap(K+m)$ has positive Lebesgue measure.

(2) $K \cap(K+m)$ consists of distinct representative $\left(\bmod \mathbb{Z}^{d}\right)$.

(3) $K \cap(K+m)$ is a packing by $\mathbb{Z}^{d}$, i.e.

$$
\sum_{n \in \mathbb{Z}^{d}} \chi_{K \cap(K+m)}(x+n) \leq 1 \text {. a.e. }
$$

Proof. Put $Q:=[0,1)^{d}$. The identity (4.1) means that for a.e. $x \in Q$, there are exactly $N$ integers $n_{1}, \ldots, n_{N}$ such that $x+n_{i} \in K i=1, \ldots, N$. Using this observation we will decompose $K$ as follows. For each $x \in Q$, put

$$
K(x):=\left\{k \in \mathbb{Z}^{d}: x+k \in K\right\} .
$$

Then $|K(x)|=N$. Let $S \subset \mathbb{Z}^{d}$ and $|S|=N$. Define

$$
K_{S}=\{x \in Q: K(x)=S\} .
$$

Since $K$ is a multi-tile of level $N$, we have

$$
K=\bigcup_{|S|=N}\left(K_{S}+S\right), \text { and } \bigcup_{|S|=N} K_{S}=Q=[0,1)^{d},
$$

where the union runs through all possible subsets $S \in \mathbb{Z}^{d}$ of cardinality $N$. Furthermore, $K_{S} \cap K_{S^{\prime}}=\emptyset, \forall S \neq S^{\prime}$, since there are exactly $N$ integers $n$ for which $x+n \in K$. By the boundedness of $K$, there are only finitely many possible $S \in \mathbb{Z}^{d}$ with $|S|=N$ such that $\left|K_{S}\right|>0$. Thus, we can enumerate those $S$ as $S_{1}, \ldots, S_{r}$ so that

$$
K=\bigcup_{i=1}^{r}\left(K_{S_{i}}+S_{i}\right) .
$$

(Notice the decomposition (4.2) also holds for any multi-tile bounded set $K$ with respect to any lattice $\Gamma$ in place of $\mathbb{Z}^{d}$ and any bounded fundamental set of $\Gamma$ in the place of $Q$.)

We order $\mathbb{Z}^{d}$ by the natural lexicographical ordering. We then enumerate all possible elements in $S_{i}, 1 \leq i \leq r$, by

$$
n_{1}^{S_{i}}<\ldots<n_{N}^{S_{i}}
$$

Let

$$
m_{S_{i}}=n_{N}^{S_{i}}-n_{1}^{S_{i}}, \text { and } m=\max _{i=1, \ldots, r} m_{S_{i}} .
$$

Note that for any $i \neq j$, since $K_{S_{i}}$ and $K_{S_{j}}$ are distinct subsets in $Q$, the intersection of $K_{S_{i}}+\mathbb{Z}^{d}$ and $K_{S_{j}}+\mathbb{Z}^{d}$ has zero Lebesgue measure. As $K_{S_{i}}+S_{i} \subset K_{S_{i}}+\mathbb{Z}^{d}$ and $K_{S_{j}}+S_{j}+m \subset K_{S_{j}}+\mathbb{Z}^{d}$, 
the set $\left(K_{S_{i}}+S_{i}\right) \cap\left(K_{S_{j}}+S_{j}+m\right)$ has zero Lebesgue measure for $i \neq j$. Therefore, up to Lebesgue measure zero, we have

$$
\begin{aligned}
K \cap(K+m) & =\left(\bigcup_{i=1}^{r}\left(K_{S_{i}}+S_{i}\right)\right) \cap\left(\bigcup_{j=1}^{r}\left(K_{S_{j}}+S_{j}+m\right)\right) \\
& =\bigcup_{i=1}^{r}\left(K_{S_{i}}+S_{i}\right) \cap\left(K_{S_{i}}+S_{i}+m\right)
\end{aligned}
$$

Note that $\left(K_{S_{i}}+S_{i}\right) \cap\left(K_{S_{i}}+S_{i}+m\right)$ has positive Lebesgue measure if and only if $S_{i} \cap\left(S_{i}+m\right) \neq \emptyset$. By our construction of $m$,

$$
S_{i} \cap\left(S_{i}+m\right)= \begin{cases}\emptyset, & \text { if } m_{S_{i}}<m \\ n_{N}^{S_{i}}, & \text { if } m_{S_{i}}=m\end{cases}
$$

Hence,

$$
K \cap(K+m)=\bigcup_{\left\{i: m_{S_{i}}=m\right\}}\left(K_{S_{i}}+n_{N}^{S_{i}}\right) .
$$

Note that $\left\{i: m_{S_{i}}=m\right\}$ is non-empty by our construction, thus $K \cap(K+m)$ has positive Lebesgue measure. On the other hand, $K_{S_{i}}$ consists of distinct representatives in $Q$. Therefore $K \cap(K+m)$ has distinct representatives $\left(\bmod \mathbb{Z}^{d}\right)$ in $Q$. This implies $(2)$. The conclusion (3) follows directly from (2) and by the definition of packing.

We remark that Proposition 4.1 does not hold if $K$ is unbounded (see Example 6.3). The following well-known lemma also hold (see [10, Lemma 2.10]).

Lemma 4.2. Let $B$ be an $d \times d$ invertible matrix and $\Omega \subset \mathbb{R}^{d}$ be a measurable set with positive and finite measure. The set $\left\{e^{2 \pi i\langle B n, x\rangle}: n \in \mathbb{Z}^{d}\right\}$ is complete in $L^{2}(\Omega)$ if and only if $\Omega$ is a packing by $B^{-t} \mathbb{Z}^{d}$, i.e.

$$
\sum_{n \in \mathbb{Z}^{d}} \chi_{\Omega}\left(x+B^{-t} n\right) \leq 1 \text {. a.e. } x \in B^{-t}(Q) .
$$

Here, $Q=[0,1)^{d}$.

We are now ready to prove Theorem 1.4.

Proof of Theorem 1.4. As we mentioned above, by Lemma 2.1 and the assumption that $A^{t} B$ is an integral matrix with $\operatorname{det}\left(A^{t} B\right)=1$, it is sufficient to prove the theorem for lattices in the form of

$$
\Lambda=\left(\begin{array}{cc}
I & O \\
C & I
\end{array}\right)\left(\mathbb{Z}^{2 d}\right)
$$

In this case, by the orthogonality of $\mathcal{G}\left(|K|^{-1 / 2} \chi_{K}, \Lambda\right)$, the exponentials $\left\{e^{2 \pi i\langle n, x\rangle}: n \in \mathbb{Z}^{d}\right\}$ are mutual orthogonal in $L^{2}(K)$. Thus, by Theorem $1.3, K=\bigcup_{j=1}^{N} E_{j}$, where $E_{j}$ 's are fundamental domains of $\mathbb{Z}^{d}$.

We claim that $N=1$. Suppose that $N>1$. By Proposition 4.1, there exists $m \in \mathbb{Z}^{d}$ such that $K \cap(K+m)$ has positive Lebesgue measure and $K \cap(K+m)$ is a packing in $\mathbb{R}^{d}$ by $\mathbb{Z}^{d}$. Thus by Lemma 4.2 , the set $\left\{e^{2 \pi i\langle n, x\rangle}: n \in \mathbb{Z}^{d}\right\}$ is complete in $K \cap(K+m)$. By Remark 2.7, $\mathbb{Z}^{d}$ is exponentially complete in $L^{2}(K \cap(K+m))$. Obviously, $m=0$ cannot satisfy the packing 
property (3) in Proposition 4.1 since we have assumed $N>1$. Thus $m \neq 0$. On the other hand, the orthogonality of the Gabor system implies that for any $n \in \mathbb{Z}^{d}$ we must have

$$
\chi_{K \cap(K+m)}(C m+n)=\int_{\mathbb{R}^{d}} \chi_{K}(x) \chi_{K}(x-m) e^{-2 \pi i\langle C m, x\rangle} e^{-2 \pi i\langle n, x\rangle} d x=0
$$

for all $n \in \mathbb{Z}^{d}$. This contradicts the exponential completeness of $\mathbb{Z}^{d}$. Therefore, the assumption $N>1$ cannot hold, and $K$ is thus a fundamental domain of $\mathbb{Z}^{d}$. This completes the proof.

Remark 4.3. In proving Theorem 1.4, once $\Lambda$ is reduced to the lattice

$$
\mathcal{A}\left(\mathbb{Z}^{2 d}\right) \text {, where } \mathcal{A}=\left(\begin{array}{cc}
I & O \\
C & I
\end{array}\right)
$$

one may suspect that we can apply a metaplectic transformation to further reduce the lattice to the separable lattice $\mathbb{Z}^{d} \times \mathbb{Z}^{d}$ and hence the problem is trivially solved. Unfortunately, this approach does not seem to work. To be precise, one can look up [13] for the metaplectic transformation in this case. We have that $\mathcal{G}\left(|K|^{-1 / 2} \chi_{K}, \mathcal{A}\left(\mathbb{Z}^{2 d}\right)\right)$ is a Gabor orthonormal basis if and only if $\mathcal{G}\left(\tilde{g}, \mathbb{Z}^{d} \times \mathbb{Z}^{d}\right)$ is a Gabor orthonormal basis, where

$$
\tilde{g}=e^{-\pi i\langle x, C x\rangle}|K|^{-1 / 2} \chi_{K} .
$$

Although the lattice is separable, the window funciton $\tilde{g}$ is now complex-valued, we cannot conclude that $K$ has no overlap in the time domain as it were the case when $C=O$.

Nonetheless, metaplectic transformation and symplectic matrices seems to provide some strong tools that may lead to a progess in the Fuglede-Gabor problem, readers may refer to $[8,12,13]$ for details about these tools.

Theorem 1.5 is now straightforward. We prove it here for the sake of completeness.

Proof of Theorem 1.5. Let $\Lambda=M\left(\mathbb{Z}^{2}\right)$ where $M=\left(\begin{array}{ll}a & d \\ c & b\end{array}\right)$ is a rational matrix with $\operatorname{det} M=$ 1. Let $q$ be the least common multiple of $a, b, c$ and $d$. Then we can write $\Lambda$ as $\Lambda=q^{-1} \tilde{M}\left(\mathbb{Z}^{2}\right)$ where $\tilde{M}$ is an integer matrix. By Lemma 2.4, we can find a unimodular integer matrix $P$ such that $\tilde{M} P$ is the lower triangular integer matrix. By the unimodularity of the matrix $P$ we have $q^{-1} \tilde{M}\left(\mathbb{Z}^{2}\right)=q^{-1} \tilde{M} P\left(\mathbb{Z}^{2}\right)$. Therefore, $\Lambda=q^{-1} \tilde{M} P\left(\mathbb{Z}^{2}\right)$ and $q^{-1} \tilde{M} P$ is a lower triangular rational matrix. We can therefore write

$$
\Lambda=\left(\begin{array}{ll}
\alpha & 0 \\
\gamma & \beta
\end{array}\right)\left(\mathbb{Z}^{2}\right)
$$

for some $\alpha, \beta, \gamma \in \mathbb{Q}$. Notice that the density of $\Lambda$ equals 1 , meaning that $\alpha \beta=1$. Thus, all assumptions of Theorem 1.4 are satisfied. Hence, $K$ is a translational tile with tiling set $\mathbb{Z}$ and is a spectral set.

\section{Proof of Theorem 1.6 - Upper triangular matrices}

We will discuss a case of upper triangular matrices which can be converted into the lower one. Then we will use Theorem 1.4 to prove the theorem. First we need few lemmas.

Lemma 5.1. Let $D$ be a $d \times d$ rational matrix. Then there is an integer lattice $\Gamma$ such that $D \gamma \in \mathbb{Z}^{d}$ for $\gamma \in \Gamma$. 
Proof. Define

$$
\Gamma:=\left\{k \in \mathbb{Z}^{d}: D k \in \mathbb{Z}^{d}\right\} .
$$

It is easy to check that $\Gamma$ is a lattice contained in $\mathbb{Z}^{d}$. Moreover, $\Gamma$ contains $p \mathbb{Z}^{d}$ where $p$ is the least common multiple of the denominators of entries of $D$. Thus, $\Gamma$ is a full-rank lattice and has the form of $\Gamma=M\left(\mathbb{Z}^{d}\right)$, for some invertible $d \times d$ matrix $M$ with integer entries.

Observe that according to the Lemma 5.1, for any given rational matrix $D$, there is an integer $M$ such that $D M$ is integer. With this observation, we have the following result.

Lemma 5.2. Let $D$ be a rational matrix, and let $\Gamma$ and $M$ be given as in Lemma 5.1 and $\operatorname{det} M=n$. Suppose that $\left\{\gamma_{1}, \cdots, \gamma_{n}\right\}$ be a complete representative $\left(\bmod M\left(\mathbb{Z}^{d}\right)\right)$ in $\mathbb{Z}^{d}$. If $D$ is symmetric, then $\left\{M^{t} D \gamma_{1}, \cdots, M^{t} D \gamma_{n}\right\}$ is a complete representative $\left(\bmod M^{t} \mathbb{Z}^{d}\right)$ in $\mathbb{Z}^{d}$.

Proof. We saw above that by the structure of $M$ and $\Gamma, D M$ is an integer matrix, therefore $D M\left(\mathbb{Z}^{d}\right) \subseteq \mathbb{Z}^{d}$. We also have $(D M)^{t} \mathbb{Z}^{d} \subseteq \mathbb{Z}^{d}$. This implies that

$$
M^{t} D\left(\mathbb{Z}^{d}\right)=\left(D^{t} M\right)^{t}\left(\mathbb{Z}^{d}\right) \subseteq \mathbb{Z}^{d} .
$$

Thus, $M^{t} D \gamma_{i}$ are all integer vectors for all $i=1, \cdots, n$.

Next, we show that $\left\{M^{t} D \gamma_{1}, \ldots, M^{t} D \gamma_{n}\right\}$ consists of distinct representative $\left(\bmod M^{t}\left(\mathbb{Z}^{d}\right)\right)$ in $\mathbb{Z}^{d}$. Suppose that this is not the case. Then for some $i \neq j$ we must have $M^{t} D \gamma_{i}-M^{t} D \gamma_{j} \in M^{t}\left(\mathbb{Z}^{d}\right)$. This implies that $D \gamma_{i}-D \gamma_{j} \in \mathbb{Z}^{d}$, which means that $\gamma_{i}$ and $\gamma_{j}$ belong to the same representative class $\left(\bmod M\left(\mathbb{Z}^{d}\right)\right)$ in $\mathbb{Z}^{d}$ which is a contradiction.

Finally, we show that $\left\{M^{t} D \gamma_{1}, \ldots M^{t} D \gamma_{n}\right\}$ is complete. This follows immediately by counting the number of cosets present in $\mathbb{Z}^{d} / M^{t}\left(\mathbb{Z}^{d}\right)$ which is $\left|\operatorname{det}\left(M^{t}\right)\right|=|\operatorname{det}(M)|=n$. Hence, $\left\{M^{t} D \gamma_{1}, \ldots M^{t} D \gamma_{n}\right\}$ is complete.

In the following constructive lemma we shall present a class of upper triangle lattice which can be converted into a lower triangular lattice.

Lemma 5.3. For $\Lambda=\left(\begin{array}{cc}I & D \\ O & I\end{array}\right)\left(\mathbb{Z}^{2 d}\right)$ with $D$ rational and symmetric, there are integer matrices $E$ and $X$ such that $\Lambda=\left(\begin{array}{cc}E^{-t} & O \\ X & E\end{array}\right)\left(\mathbb{Z}^{2 d}\right)$.

Proof. Let $\left\{\mathbf{e}_{\mathbf{i}}: i=1, \cdots, d\right\}$ denote the standard basis in $\mathbb{Z}^{d}$. Associated to this $D$ let $\Gamma$ and $M$ be given as in Lemma 5.1. Then by Lemma 5.2, for any $i$ there exists $z_{i} \in \mathbb{Z}^{d}$ and $\gamma^{i} \in$ $\left\{\gamma_{1}, \cdots, \gamma_{n}\right\}$ such that $\mathbf{e}_{\mathbf{i}}=M^{t} z_{i}+M^{t} D \gamma^{i}$. Put $Z:=\left[z_{1} \cdots z_{d}\right]$ and $X=\left[\gamma_{1} \cdots \gamma_{d}\right]$. It is clear that $Z$ and $X$ are integer matrices. A direct calculation shows that $(Z+D X)\left(\mathbb{Z}^{d}\right)=M^{-t}\left(\mathbb{Z}^{d}\right)$.

Put $P:=\left(\begin{array}{cc}Z & -D M \\ X & M\end{array}\right)$. Then $P$ is an integer matrix with $\operatorname{det} P=1$ and $P\left(\mathbb{Z}^{2 d}\right)=\mathbb{Z}^{2 d}$. Recall that $D M$ is an integer matrix. So we can write

$$
\Lambda=\left(\begin{array}{cc}
I & D \\
O & I
\end{array}\right)\left(\mathbb{Z}^{2 d}\right)=\left(\begin{array}{cc}
I & D \\
O & I
\end{array}\right) P\left(\mathbb{Z}^{2 d}\right)=\left(\begin{array}{cc}
M^{-t} & O \\
X & M
\end{array}\right)\left(\mathbb{Z}^{2 d}\right) .
$$

Now take $E=M$, and we are done. 
At this point we are ready to complete the proof of Theorem 1.6.

Proof of Theorem 1.6. Let $\Lambda=\left(\begin{array}{cc}A & D \\ O & B\end{array}\right)\left(\mathbb{Z}^{2 d}\right)$ where $B=A^{-t}$ and $\widetilde{D}:=A^{-1} D$ is rational and symmetric. By Lemma 2.1, the associated matrix can be reduced to a block matrix of the form $\left(\begin{array}{cc}I & \widetilde{D} \\ O & I\end{array}\right)$ where $\widetilde{D}$ is rational and symmetric. Therefore, it is sufficient to prove the theorem for a lattice of the form $\Lambda=\left(\begin{array}{cc}I & D \\ O & I\end{array}\right)\left(\mathbb{Z}^{2 d}\right)$ where $D$ is rational and symmetric. By Lemma 5.3, there are integer matrices $E$ and $X$ such that $\Lambda=\left(\begin{array}{cc}E^{-t} & O \\ X & E\end{array}\right)\left(\mathbb{Z}^{2 d}\right)$. The rest of the proof is now a conclusion of Theorem 1.4 and we have completed the proof.

In [16], the authors proved that for any lattice $\Lambda$ formed by an upper triangular matrix exists a window $g$ such that $\mathcal{G}(g, \Lambda)$ is a Gabor orthonormal basis and such $g$ satisfies $\widehat{g}=\chi_{K}$, where $K$ is the common fundamental domain for the diagonal block matrices $A$ and $B^{-t}$. However, their proof does not provide any constructive technique for producing a compactly supported window. The proof of Theorem 1.6 provides a technique to construct a large class of examples of sets $K$ forming a Gabor orthonormal basis with respect to the lattice generated by upper triangular matrices. We explain this next.

Proposition 5.4. Assume that the lattice $\Lambda$ and matrices $A, B$ and $D$ are given as in Theorem 1.6 and satisfying the hypotheses of the theorem. Then there is a set $K$ such that $\mathcal{G}\left(|K|^{-1 / 2} \chi_{K}, \Lambda\right)$ is a Gabor orthonormal basis for $L^{2}\left(\mathbb{R}^{d}\right)$.

Proof. Let $A, B$ and $D$ be given. Notice that by Lemma 2.1 and the hypotheses of the proposition we know that for any given set $K$, the system $\mathcal{G}\left(|K|^{-1 / 2} \chi_{K}, \Lambda\right)$ is an orthonormal basis if and only if $\mathcal{G}\left(\left|A^{-1}(K)\right|^{-1 / 2} \chi_{A^{-1}(K)}, \tilde{\Lambda}\right)$ is an orthonormal basis where $\tilde{\Lambda}=\left(\begin{array}{cc}I & A^{-1} D \\ O & I\end{array}\right)\left(\mathbb{Z}^{2 d}\right)$. And, by Lemma 5.3 we also know that for $\tilde{\Lambda}$ there are integer matrices $X$ and $E$ such

$$
E\left(\mathbb{Z}^{d}\right)=\left\{n \in \mathbb{Z}^{d}: A^{-1} D n \in \mathbb{Z}^{d}\right\}
$$

and $\widetilde{\Lambda}=\left(\begin{array}{cc}E^{-t} & O \\ X & E\end{array}\right)\left(\mathbb{Z}^{2 d}\right)$. By appealing to Lemma 2.1 one more time, $\mathcal{G}\left(|K|^{-1 / 2} \chi_{K}, \Lambda\right)$ is an orthonormal basis if and only if

$$
\mathcal{G}\left(|K|^{-1 / 2} \chi_{K},\left(\begin{array}{cc}
A E^{-t} & O \\
A^{-t} X & A^{-t} E
\end{array}\right)\left(\mathbb{Z}^{2 d}\right)\right)
$$

is an orthonormal basis. Now take $K$ as a fundamental domain of $A E^{-t}\left(\mathbb{Z}^{d}\right)$ and we are done.

The following gives a more explicit example.

Example 5.5. Let $A=\left(\begin{array}{cc}\frac{1}{2} & 0 \\ 0 & 2\end{array}\right), B=\left(\begin{array}{cc}2 & 0 \\ 0 & \frac{1}{2}\end{array}\right)$. Take $D=\left(\begin{array}{cc}1 & 0 \\ 0 & \frac{1}{3}\end{array}\right)$. Then $A^{-1} D$ is rational and symmetric. Let $\Gamma=\left\{n \in \mathbb{Z}^{2}: A^{-1} D n \in \mathbb{Z}^{2}\right\}$. $\Gamma$ is a full lattice and a simple calculation shows that $\Gamma=E\left(\mathbb{Z}^{2}\right)$ where $E=\left(\begin{array}{ll}1 & 0 \\ 0 & 6\end{array}\right)$. Now let $K$ be any fundamental 
domain of the lattice $A E^{-t}\left(\mathbb{Z}^{2}\right)=\left(\begin{array}{cc}1 / 2 & 0 \\ 0 & 1 / 3\end{array}\right)\left(\mathbb{Z}^{2}\right)$. Then by Proposition 5.4 the system $\mathcal{G}\left(|K|^{-1 / 2} \chi_{K}, \Lambda\right)$ is a Gabor orthonormal basis for $L^{2}\left(\mathbb{R}^{2}\right)$ for the lower triangular lattice $\Lambda$ with diagonal matrices $A E^{-t}$ and $A^{-t} E$ and any matrix $C$.

\section{EXAMPLES}

Consider $\Lambda=L\left(\mathbb{Z}^{2 d}\right)$ where $L=\left(\begin{array}{cc}A & O \\ C & B\end{array}\right)$ is rational. In Theorem 1.4 we proved that for a set $K$ if $\mathcal{G}\left(|K|^{-1 / 2} \chi_{K}, \Lambda\right)$ is an orthonormal basis for $L^{2}\left(\mathbb{R}^{d}\right)$ and $A^{t} B$ is an integer matrix, then $K$ is a fundamental domain of $A\left(\mathbb{Z}^{d}\right)$. By Theorem 1.3 this means $N=1$. Thus $K$ tiles by $A\left(\mathbb{Z}^{d}\right)$ and is spectral with spectrum $B^{-t}\left(\mathbb{Z}^{d}\right)$. In this section we will provide examples showing that $N>1$ can also happen in Theorem 1.3 if $A^{t} B$ is not an integer matrix and yet the system $\mathcal{G}\left(|K|^{-1 / 2} \chi_{K}, \Lambda\right)$ is an orthonormal basis for $L^{2}\left(\mathbb{R}^{d}\right)$.

We are now ready to present our example of a set $K$ which is the union of fundamental domains of lattice $A\left(\mathbb{Z}^{d}\right)$ and the union of fundamental domains of lattice $B^{-t}\left(\mathbb{Z}^{d}\right), \chi_{K}$ is a window function for a possible Gabor orthonormal basis, and $A^{t} B$ is not an integer matrix.

Example 6.1. Let $K=[0,2] \times[0,1]$ and $\Lambda=\left(\begin{array}{cc}I & O \\ C & B\end{array}\right)\left(\mathbb{Z}^{4}\right)$ where $B=\left(\begin{array}{cc}1 / 2 & 0 \\ 0 & 2\end{array}\right)$ and $C=\left(\begin{array}{ll}c_{11} & c_{12} \\ c_{21} & c_{22}\end{array}\right)$. The system $\mathcal{G}\left(|K|^{-1 / 2} \chi_{K}, \Lambda\right)$ is a Gabor orthonormal basis if $c_{21}$ is an odd number.

Proof. Observe that

$$
\begin{aligned}
{[0,2] \times[0,1] } & =[0,1]^{2}+\{(0,0),(1,0)\} \\
& =[0,2] \times[0,1 / 2]+\{(0,0),(0,1 / 2)\}
\end{aligned}
$$

This shows that $K$ is union of two fundamental domains of $\mathbb{Z}^{2}$ and union of two fundamental domains of $B^{-t}\left(\mathbb{Z}^{2}\right)$, respectively.

Let $(m, n) \in \mathbb{Z}^{4}$ with $m, n \in \mathbb{Z}^{2}$ and $(m, n) \neq(0,0)$. Put

$$
\begin{aligned}
\mathcal{I} & :=\int \chi_{K}(x-m) \chi_{K}(x) e^{-2 \pi i\langle C(m), x\rangle} e^{-2 \pi i\langle B(n), x\rangle} d x \\
& =\int \chi_{(K+\mathbf{m}) \cap K}(x) e^{-2 \pi i\langle C(m), x\rangle} e^{-2 \pi i\langle B(n), x\rangle} d x .
\end{aligned}
$$

Mutual orthogonality of the Gabor system will follow if we can show that $\mathcal{I}=0$ for any $(m, n) \neq$ $(0,0)$. Note that

$$
(K+m) \cap K \simeq \begin{cases}K, & m=0 \\ {[0,1]^{2},} & m=( \pm 1,0) \\ \emptyset, & \text { otherwise }\end{cases}
$$

(Here, we write $A \simeq B$ if $A=B+k$ for some $k \in \mathbb{Z}^{d}$.)

Since $K$ is a union of fundamental domains for $B^{-t}\left(\mathbb{Z}^{2}\right)$, then if $m=0$, we automatically have $\mathcal{I}=0$. 
If $m=( \pm 1,0)$, for any $n=\left(n_{1}, n_{2}\right), \mathcal{I}$ is equal to the following integral up to a unimodular constant:

$$
\begin{aligned}
\int_{[0,1]^{2}} e^{-2 \pi i\left\langle C\left(( \pm 1,0)^{t}\right), x\right\rangle} e^{-2 \pi i\langle B(n), x\rangle} d x & =\int_{[0,1]} \int_{[0,1]} e^{-2 \pi i\left( \pm c_{11} x_{1} \pm c_{21} x_{2}\right)} e^{-2 \pi i\left(\frac{1}{2} n_{1} x_{1}+2 n_{2} x_{2}\right)} d x_{1} d x_{2} \\
& =\widehat{\chi_{[0,1]}}\left( \pm c_{11}+\frac{1}{2} n_{1}\right) \widehat{\chi_{[0,1]}}\left( \pm c_{21}+2 n_{2}\right) .
\end{aligned}
$$

It is obvious that the last line equals to zero for all $\left(n_{1}, n_{2}\right)$ only if $c_{21}$ is an odd number. For other cases of $m$ it is trivial that $\mathcal{I}=0$. Thus the orthogonality is obtained and the completeness is a direct conclusion of Proposition 2.2.

We notice that the previous example exploited the fact that $B\left(\mathbb{Z}^{2}\right)$ is exponentially incomplete for $L^{2}[0,1]^{2}$ in (6.1). The following example illustrates a case where, contrary to the previous example, the finite union of fundamental domains cannot form a Gabor orthonormal basis for any choice of matrix $C$.

Example 6.2. Let $K=[0,2]^{2}$ and let $B$ be the matrix as in Example 6.1. Then $K$ is union of 4 fundamental domains of the lattice $\mathbb{Z}^{2}$ and union of 4 fundamental domains of $B^{-t}\left(\mathbb{Z}^{2}\right)$. However, there is no matrix $C$ for which $\chi_{K}$ is a window function yielding an orthonormal basis for the lower triangular lattice $\Lambda=\left(\begin{array}{cc}I & O \\ C & B\end{array}\right)\left(\mathbb{Z}^{4}\right)$.

Proof. The fact that $K$ is union of fundamental domains of $\mathbb{Z}^{d}$ and $B^{-t}\left(\mathbb{Z}^{d}\right)$ is straight forward. In short,

$$
\begin{aligned}
K & =[0,1]^{2}+\{(0,0),(1,0),(0,1),(1,1)\} \\
& =[0,2] \times[0,1 / 2]+\{(0,0),(0,1 / 2),(0,1),(0,3 / 2)\} .
\end{aligned}
$$

Suppose that there exists a matrix $C$ such that $\chi_{K}$ is a window function for the lower triangular lattice $\Lambda=\left(\begin{array}{cc}I & O \\ C & B\end{array}\right)\left(\mathbb{Z}^{4}\right)$. Thus, for any $(0,0) \neq(m, n) \in \mathbb{Z}^{4}$ we must have

$$
0=\mathcal{I}:=\int \chi_{(K+m) \cap K}(x) e^{-2 \pi i\langle C(m), x\rangle} e^{-2 \pi i\langle B(n), x\rangle} d x .
$$

From the other side, the only non-empty intersection sets $(K+m) \cap K$ are

$$
(K+m) \cap K \simeq \begin{cases}K, & \text { if } m=0 \\ {[0,1] \times[0,2],} & \text { if } m=( \pm 1,0) \\ {[0,2] \times[0,1],} & \text { if } m=(0, \pm 1) \\ {[0,1]^{2},} & \text { if } m=( \pm 1, \pm 1)\end{cases}
$$

From the last three intersections, we obtain that if $\mathcal{I}=0$, then the following three equations must hold for all integer vectors $\left(n_{1}, n_{2}\right)$, respectively:

$$
\begin{array}{r}
\widehat{\chi_{[0,1]}}\left( \pm c_{11}+\frac{1}{2} n_{1}\right) \widehat{\chi_{[0,2]}}\left( \pm c_{21}+2 n_{2}\right)=0 \\
\widehat{\chi_{[0,2]}}\left( \pm c_{12}+\frac{1}{2} n_{1}\right) \widehat{\chi_{[0,1]}}\left( \pm c_{22}+2 n_{2}\right)=0 \\
\widehat{\chi_{[0,1]}}\left( \pm c_{11} \pm c_{12}+\frac{1}{2} n_{1}\right) \widehat{\chi_{[0,1]}}\left( \pm c_{21} \pm c_{22}+2 n_{2}\right)=0
\end{array}
$$


We claim that if the first equation holds, then $\widehat{\chi_{[0,2]}}\left( \pm c_{21}+2 n_{2}\right)=0$ for all integers $n_{2}$ and $c_{21} \in 2 \mathbb{Z}+\left\{\frac{1}{2}, 1, \frac{3}{2}\right\}$.

To justify the claim, suppose that there exists an integer $n_{2}$ such that $\widehat{\chi_{[0,2]}}\left( \pm c_{21}+2 n_{2}\right) \neq 0$. Then $\widehat{\chi_{[0,1]}}\left( \pm c_{11}+\frac{1}{2} n_{1}\right)=0$ for all integers $n_{1}$. However, this would imply the existence of an exponentials $e^{-2 \pi i c_{11} x}$ such that it is orthogonal to all $\left\{e^{2 \pi i\left(\frac{1}{2} n\right) x}: n \in \mathbb{Z}\right\}$ in $L^{2}[0,1]$. This is impossible since the exponentials set $\left\{e^{2 \pi i\left(\frac{1}{2} n\right) x}: n \in \mathbb{Z}\right\}$ is complete in $L^{2}[0,1]$. Hence, we have only $\widehat{\chi_{[0,2]}}\left( \pm c_{21}+2 n_{2}\right)=0$ for all integers $n_{2}$. Finally, since the zero set for $\widehat{\chi_{[0,2]}}$ is $\frac{1}{2} \mathbb{Z}$ except zero, then we must have $c_{21} \in 2 \mathbb{Z}+\left\{\frac{1}{2}, 1, \frac{3}{2}\right\}$, as desired.

Similarly, the second equation implies that $\widehat{\chi_{[0,1]}}\left( \pm c_{22}+2 n_{2}\right)=0$ for all integers $n_{2}$ and thus $c_{22} \in 2 \mathbb{Z}+1$ must be an odd integer.

The third equation implies that

$$
\widehat{\chi_{[0,1]}}\left( \pm c_{21} \pm c_{22}+2 n_{2}\right)=0
$$

for any integers $n_{2}$. Now, we write $c_{21}=2 k_{1}+\frac{1}{2} j$, for some $j=1,2,3$ and $c_{22}=2 k_{2}+1$. Then $\pm c_{21} \pm c_{22} \in 2 \mathbb{Z}+\left\{\frac{3}{2}, 2, \frac{5}{2}\right\}$. In the case of fraction, (6.2) cannot be zero. If $\pm c_{21} \pm c_{22}=2 m+2$, we take $n_{2}=-m-1$. Then $(6.2)$ will imply $\widehat{\chi_{[0,1]}}(0)=1$, which is impossible. Thus, the third equation can never be zero. This implies that such $C$ does not exist.

The following example proves that the boundedness property for the set $K$ is necessary in Proposition 4.1.

Example 6.3. Let $I_{0}=[0,1)$ and $I_{n}=\left[1-\frac{1}{2^{n-1}}, 1-\frac{1}{2^{n}}\right)$ for $n \geq 1$. Define

$$
K=\bigcup_{k \in \mathbb{Z}}\left(k+I_{|k|}\right)
$$

The set $K$ is unbounded and we have the following.

(1) $K$ multi-tiles $\mathbb{R}$ by $\mathbb{Z}$ at level 3 . However, for any $m \neq 0, K \cap(K+m)$ is not a packing of $\mathbb{R}$. Therefore, Proposition 4.1 does not hold if $K$ is unbounded.

(2) Nonetheless, $K$ cannot form a Gabor orthonormal basis using lattices of the form $\left(\begin{array}{ll}1 & 0 \\ c & 1\end{array}\right)\left(\mathbb{Z}^{2}\right)$

Proof. The fact that $K$ mult-tiles $\mathbb{R}$ by $\mathbb{Z}$ at level 3 follows from a direct observation, so we omit the details here. We note that for any $m \neq 0$,

$$
K \cap(K+m) \supset I_{|m|} \cup\left(I_{|m|}+m\right) .
$$

Hence, for all $x \in I_{|m|}, \sum_{n \in \mathbb{Z}} \chi_{K \cap(K+m)}(x+n) \geq 2$. Therefore, it is never a packing for any $m \neq 0$. To show the last statement, notice

$$
K \cap(K+1)=\left[0, \frac{1}{2}\right) \cup\left[1, \frac{3}{2}\right), K \cap(K+2)=\left[\frac{1}{2}, \frac{3}{4}\right) \cup\left[1, \frac{3}{2}\right) \cup\left[\frac{5}{2}, \frac{11}{4}\right)
$$


Suppose that $K$ forms a Gabor orthonormal basis using some lattice of the form $\left(\begin{array}{ll}1 & 0 \\ c & 1\end{array}\right)\left(\mathbb{Z}^{2}\right)$. Then $\chi_{K \cap(K+1)}(c+m)=0$ and $\chi_{K \cap(K+2)}(c+m)=0$ for all $m \in \mathbb{Z}$. In particular,

$$
\widehat{\chi \cap \cap(K+1)}(c+m)=\left(1+e^{2 \pi i(c+m)}\right) \widehat{\chi_{[0,1 / 2]}}(c+m)=0, \forall m \in \mathbb{Z} .
$$

We see that the only possibility of the above is that $c \in \frac{1}{2}+\mathbb{Z}$. We now consider

$$
\chi_{\overline{K \cap(K+2)}}(\xi)=e^{2 \pi i \frac{1}{2} \xi}\left(1+e^{2 \pi i \frac{1}{2} \xi}+e^{2 \pi i \frac{3}{4} \xi}+e^{2 \pi i \frac{2}{\xi}}\right) \widehat{\chi_{[0,1 / 4)}}(\xi) .
$$

If $c \in \frac{1}{2}+\mathbb{Z}$ and $\widehat{\chi K \cap(K+2)}(c)=0$, we must have

$$
0=1+e^{2 \pi i \frac{1}{2} c}+e^{2 \pi i \frac{3}{4} c}+e^{2 \pi i 2 c}=2+e^{2 \pi i \frac{1}{2} c}+e^{2 \pi i \frac{3}{4} c} .
$$

This forces $e^{2 \pi i \frac{1}{2} c}=e^{2 \pi i \frac{3}{4} c}=-1$. Thus,

$$
c \in 2(1 / 2+\mathbb{Z}) \cap \frac{4}{3}(1 / 2+\mathbb{Z})=(1+2 \mathbb{Z}) \cap\left(\frac{2}{3}+\frac{4}{3} \mathbb{Z}\right) \subset 1+2 \mathbb{Z}
$$

This is a contradiction since $c \in \frac{1}{2}+\mathbb{Z}$ is never an integer. This completes the proof.

\section{Discussions AND Open PROBlems}

This paper investigates the Fuglede-Gabor Problem 1.2 over the lattices. We believe that this problem should be true for all lattices. We solved the problem completely in dimension one when the lattice is rational and in higher dimensions when the lattice is integer. In what follows we shall explain how to resolve Problem 1.2 in full generality for any lattices $\Lambda=\left(\begin{array}{cc}A & D \\ C & B\end{array}\right)\left(\mathbb{Z}^{2 d}\right)$. In fact, it is sufficient to solve the following two cases.

(1) Rational case: After converting a rational lattice into a lower triangular rational matrix by Corollary 2.5 and reducing the matrix where $A=I$ by Lemma $2.1, \Lambda$ is a lattice of the form of $\left(\begin{array}{cc}I & O \\ C & B\end{array}\right)\left(\mathbb{Z}^{2 d}\right)$, where $B$ and $C$ are $d \times d$ rational matrices but $B$ is not necessarily an integral matrix.

(2) Irrational case: After a reduction process by Lemma 2.1, $\Lambda$ is a lattice of the form of

$$
\left(\begin{array}{ll}
I & D \\
C & B
\end{array}\right)\left(\mathbb{Z}^{2 d}\right)
$$

which contains irrational entries.

In what follows, we shall discuss these two cases in more details.

7.1. Rational Case. Let $\Lambda=\left(\begin{array}{cc}A & O \\ C & B\end{array}\right)\left(\mathbb{Z}^{2 d}\right)$ be a lower triangular rational matrix. Example 6.1 tells us that when $A^{t} B$ is a non-integer matrix, $K$ is not a fundamental domain for the lattices $A\left(\mathbb{Z}^{d}\right)$ and $B^{-t}\left(\mathbb{Z}^{d}\right)$ but the union of their fundamental domains. However, in Example 6.2 we see that there exists no $C$ such that $K$, as the union of fundamental domains, is a set whose characteristic function generates a Gabor orthonormal basis associated to the given matrices $A$ and $B$. We predict that this failure is due to the number of decompositions of $K$ into fundamental 
domains of $B^{-t}\left(\mathbb{Z}^{d}\right)$. In this concern and in relation to the examples illustrated in Section 6, we conjecture the following problem for the lattices $\Lambda=\left(\begin{array}{cc}I & D \\ C & B\end{array}\right)\left(\mathbb{Z}^{2 d}\right)$.

Conjecture 7.1. Let $K \subset \mathbb{R}^{d}$ and $B$ be a rational matrix with $\operatorname{det}(B)=1$. Let $s$ be the least common multiple of the denominators of the matrix entries $\left(b_{i j}\right)$. There is a matrix $C$ such that $\mathcal{G}\left(|K|^{-1 / 2} \chi_{K}, \Lambda\right)$ is a Gabor orthonormal basis for $L^{2}\left(\mathbb{R}^{d}\right)$ if and only if $K$ tiles and

$$
K=\bigcup_{i=1}^{s} E_{i}=\bigcup_{i=1}^{s} F_{i}
$$

where $E_{i}$ and $F_{i}$ are fundamental domains of $\mathbb{Z}^{d}$ and $B^{-t}\left(\mathbb{Z}^{d}\right)$, respectively.

It is obvious that the conjecture automatically holds when $B$ is an integer matrix which means $s=1$. Indeed, this is the result of Theorem 1.4.

Observe that if $K$ is as in Conjecture 7.1, then for any non-zero $n \in \mathbb{Z}^{d}$ we have $\int_{K} e^{2 \pi i\langle B n, x\rangle} d x=$ 0 . And, there are only finitely many $m \in \mathbb{Z}^{d}$ such that $|K \cap(K+m)| \neq 0$, as $K$ is a finite union of fundamental domains of $\mathbb{Z}^{d}$. To prove the orthogonality, one must first show the existence of a matrix $C$ such that for all $n \in \mathbb{Z}^{d}$

$$
\int_{K \cap K+m} e^{-2 \pi i\langle C m, x\rangle} e^{-2 \pi i\langle B n, x\rangle} d x=0
$$

which is equivalent to the exponential incompleteness of the lattices $B\left(\mathbb{Z}^{d}\right)$ for $L^{2}(K \cap K+m)$. It appears that we need to study the exponential completeness of the lattices over different domains. In fact, the following problem has not yet had a definite answer.

Problem 7.2. Given a set $K$ with positive and finite measure, classify all invertible $d \times d$ matrices $B$ for which the exponents $\left\{e^{-2 \pi i\langle B n, x\rangle}: n \in \mathbb{Z}^{d}\right\}$ are exponentially complete in $L^{2}(K)$.

Proposition A.2 provides a sufficient condition for matrices $B$ when $K=[0,1]^{d}$. Unfortunately, the converse of the proposition does not hold in dimensions $d=2$ and higher, as the counterexample following Proposition A.2 shows. However, in Proposition A.1 we obtain a full characterization of exponential completeness for $[0,1]$ in dimension $d=1$ for integer lattices in $\mathbb{Z}$.

As mentioned earlier, the exponential completeness of a set does not imply the completeness of the set in general. For a recent developments in study of the completeness, frame and Riesz bases properties of exponentials we refer the reader to the paper by De Carli and her co-authors [2].

7.2. Irrational Cases. The case for irrational lattices is more challenging and complicated. It appears that the lower and upper triangular case is asymmetric. We have seen that in Theorem 1.4, the lower triangular block matrix $C$ is not involved in the statement. Thus, irrational entries are allowed for lower triangular matrices. On the other hand, Han and Wang [16] implicitly conjectured the following problem in their paper [15]: 
Han and Wang's Conjecture: Let $\Lambda=\left(\begin{array}{ll}1 & \alpha \\ 0 & 1\end{array}\right)\left(\mathbb{Z}^{2}\right)$ where $\alpha$ is irrational. Then there doesn't exist compactly supported window $g$ such that $\mathcal{G}(g, \Lambda)$ forms a Gabor orthonormal basis for $L^{2}(\mathbb{R})$.

Observe that if $\Lambda=\left(\begin{array}{cc}1 & \alpha \\ 0 & 1\end{array}\right)$, with $\alpha$ irrational, our method applied to construct Example 5.5 would not work for the existence of $K$ since in that case $\Gamma=\{0\}$. This observation predicts that Han and Wang Conjecture [15] might be true, although we do not have a proof for it now. However, a simple calculation shows that the function $\chi_{[0,1]}$ can not be a window function for this lattice.

7.3. Full generality of Fuglede-Gabor Problem 1.2. It is known that non-symmetric convex bodies as well as convex sets with a point of non-vanishing Gaussian curvature have no basis of exponentials and yet they do not tile [23, 17]. Recently, similar results were also proved for Gabor bases. Indeed, the authors in [18] proved that in dimensions $d \neq 1(\bmod 4)$, convex sets with a point of non-vanishing Gaussian curvature cannot generate any Gabor orthonormal basis with respect to any countable time-frequency set $\Lambda$. Also, the authors in [4] proved that non-symmetric convex polytopes do not produce any Gabor orthonormal basis with respect to any countable time-frequency set. However, the result for non-symmetric convex domains is not known yet. The existent results predict that Fuglede-Gabor problem will still be true to some extent.

One may notice that there are also examples of spectral sets which do not tile by translations (see e.g. [28], [25] and [22]). Therefore, by a result of Dutkay and the first listed author [5], it is known that the indicator function of these sets can not serve as Gabor orthonormal window with resepct to any separable time-frequency set. However, they may still produce a Gabor orthonormal basis using some non-separable and countable time-frequency sets. This requires some input of new ideas.

Finally, it is known that octagon does not tile $\mathbb{R}^{2}$ by translations, but it is a multi-tile by $\mathbb{Z}^{2}$. The following example tells us that it does not form Gabor orthonormal basis using any lattices, confirming that the Fuglede-Gabor problem holds up to some extent.

Example 7.3. Let $\mathcal{O}_{8} \subset \mathbb{R}^{2}$ be the octagon symmetrically centred at the origin with integer vertices $\{( \pm 1, \pm 2),( \pm 2, \pm 1)\}$. $\mathcal{O}_{8}$ multi-tiles $\mathbb{R}^{2}$ with $\mathbb{Z}^{2}$ and it is the union of $s=14$ fundamental domains of $\mathbb{Z}^{2}$. Yet there doesn't exist any $\Lambda=\left(\begin{array}{cc}I & O \\ C & B\end{array}\right)\left(\mathbb{Z}^{4}\right)$ with rational matrix $B$ for which $\mathcal{G}\left(\left|\mathcal{O}_{8}\right|^{-1 / 2} \chi_{\mathcal{O}_{8}}, \Lambda\right)$ forms an orthonormal basis for $L^{2}\left(\mathbb{R}^{2}\right)$.

The proof of this example is involved and so it will be provided in the next section.

\section{Gabor orthonormal bases on Octagon and Proof of Example 7.3}

In this appendix, we will prove that the octagon symmetrically centered at the origin with integer vertices $\{( \pm 1, \pm 2),( \pm 2, \pm 1)\}$ cannot admit any Gabor orthonormal basis with $\Lambda=$ $\left(\begin{array}{ll}I & O \\ C & B\end{array}\right)\left(\mathbb{Z}^{4}\right)$, where $B$ is a rational matrix. For this we need the following lemma. 
Lemma 8.1. Let $M=\left(\begin{array}{cc}\alpha & 0 \\ 0 & 1 / \alpha\end{array}\right)$ and $M_{\beta}=\left(\begin{array}{cc}\alpha & 0 \\ \beta & 1 / \alpha\end{array}\right), \alpha \neq 0$. Then $\Omega$ is a multi-tile by $M_{\beta}\left(\mathbb{Z}^{2}\right)$ if and only if $\Omega$ is a multi-tile by $M\left(\mathbb{Z}^{2}\right)$.

Proof. Let $\alpha>0$ and put $Q_{\alpha}=[0, \alpha) \times[0,1 / \alpha)$. Then $Q_{\alpha}$ is a fundamental domain for the lattice $M\left(\mathbb{Z}^{2}\right)$. We claim that $Q_{\alpha}$ is a fundamental domain for $M_{\beta}\left(\mathbb{Z}^{2}\right)$ for any $\beta \in \mathbb{R}$. Indeed, this follows from a direct calculation: For almost every $(x, y)$, we have

$$
\begin{aligned}
\sum_{m, n \in \mathbb{Z}} \chi_{Q_{\alpha}}(x-\alpha m, y-\beta m-n / \alpha) & =\sum_{m \in \mathbb{Z}} \chi_{[0, \alpha)}(x-\alpha m)\left(\sum_{n \in \mathbb{Z}} \chi_{[0,1 / \alpha)}(y-\beta m-n / \alpha)\right) \\
& =\sum_{m \in \mathbb{Z}} \chi_{[0, \alpha)}(x-\alpha m)=1 .
\end{aligned}
$$

Let $\Omega$ be any set which is a tile by $M\left(\mathbb{Z}^{2}\right)$. Let $\left\{E_{(u, v)}:(u, v) \in \mathbb{Z}^{2}\right\}$ be a partition of $Q_{\alpha}$ such that

$$
\Omega=\bigcup_{(u, v) \in \mathbb{Z}^{2}}\left(E_{(u, v)}+(\alpha u, v / \alpha)\right)
$$

Then set $\Omega$ is a tile by $M_{\beta}\left(\mathbb{Z}^{2}\right)$. Indeed,

$$
\begin{aligned}
\sum_{m, n \in \mathbb{Z}} \chi_{\Omega}(x-\alpha m, y-\beta m-n / \alpha) & =\sum_{m, n \in \mathbb{Z}} \sum_{(u, v) \in \mathbb{Z}^{2}} \chi_{E_{(u, v)+(\alpha u, v / \alpha)}}(x-\alpha m, y-\beta m-n / \alpha) \\
& =\sum_{(u, v) \in \mathbb{Z}^{2}} \sum_{m, n \in \mathbb{Z}} \chi_{E_{(u, v)}}(x-\alpha(m+u), y-\beta m-(n+v) / \alpha) \\
& =\sum_{(u, v) \in \mathbb{Z}^{2}} \sum_{m, n \in \mathbb{Z}} \chi_{E_{(u, v)}}(x-\alpha m, y-\beta m-n / \alpha) \\
& =\sum_{m, n \in \mathbb{Z}} \sum_{(u, v) \in \mathbb{Z}^{2}} \chi_{E_{(u, v)}}(x-\alpha m, y-\beta m-n / \alpha) \\
& =\sum_{m, n \in \mathbb{Z}} \chi_{Q_{\alpha}}(x-\alpha m, y-\beta m-n / \alpha)=1 .
\end{aligned}
$$

Note that above we used an application of Fubini's theorem. Now let $\Omega$ be a multi-tile with respect to the lattice $M\left(\mathbb{Z}^{2}\right)$. Then $\Omega=\bigcup_{i=1}^{N} \Omega_{i}$, where $\Omega_{i}$ are tiles by $M\left(\mathbb{Z}^{2}\right)$. Thus, by the previous results above, each $\Omega_{i}$ is a tile by $M_{\beta}\left(\mathbb{Z}^{2}\right)$, hence we have the following.

$$
\sum_{m, n \in \mathbb{Z}} \chi_{\Omega}(x-\alpha m, y-\beta m-n / \alpha)=\sum_{i=1}^{N} \sum_{m, n \in \mathbb{Z}} \chi_{\Omega_{i}}(x-\alpha m, y-\beta m-n / \alpha)=N .
$$

The converse can be obtained by a similar calculation.

Proof of Example 7.3. Before we prove our claim, note that if the family $\mathcal{G}\left(\left|\mathcal{O}_{8}\right|^{-1 / 2} \chi_{\mathcal{O}_{8}}, \Lambda\right)$ is a Gabor basis and $B$ is an integer matrix, then according to Theorem 1.4 the set $\mathcal{O}_{8}$ must tile which is impossible. Thus, we assume that $B$ has some non-integer rational entries. Let $\mathbf{m}_{0}=(3,2)$. 
Then $\mathcal{P}:=K \cap\left(K+\mathbf{m}_{0}\right), K=\mathcal{O}_{8}$, is the parallelogram with vertices $\{(1,2),(2,1),(1,1),(2,0)\}$. Hence,

$$
\mathcal{P}=Q[0,1]^{2}+(1,1)^{t}, \text { where } Q=\left(\begin{array}{cc}
1 & 0 \\
-1 & 1
\end{array}\right) .
$$

The Fourier transform of $\chi_{\mathcal{P}}$ at $\xi=\left(\xi_{1}, \xi_{2}\right)$ is given by

$$
\widehat{\chi \mathcal{P}}(\xi)=c \widehat{\chi[0,1]^{2}}\left(Q^{T} \xi\right)
$$

where $c:=c(\xi)$ is some unimodular constant.

By the mutual orthogonality of the element $\left(m_{0}, n\right)$ with $(0,0)$ for all $n \in \mathbb{Z}^{2}$, we obtain

$$
0=I:=\int_{K \cap\left(K+m_{0}\right)} e^{-2 \pi i\left\langle C m_{0}, x\right\rangle} e^{-2 \pi i\langle B n, x\rangle} d x=c \cdot \widehat{\chi_{[0,1]^{2}}}\left(Q^{T} C m_{0}+Q^{T} B n\right) .
$$

If $C m_{0}=0$, by putting $n=0$, then one must have $c=0$, which is a contradiction . Otherwise, (8.1) shows that $Q^{T} B\left(\mathbb{Z}^{2}\right)$ is exponentially incomplete for $L^{2}[0,1]^{2}$. By Theorem A.3 in the appendix, $Q^{T} B$ is equivalent to one of the following two forms.

$$
\left(\begin{array}{cc}
\frac{1}{q^{\prime}} & 0 \\
r^{\prime} & q^{\prime}
\end{array}\right) \text { or }\left(\begin{array}{cc}
p^{\prime} & 0 \\
\frac{r^{\prime \prime}}{s^{\prime \prime}} & \frac{1}{p^{\prime}}
\end{array}\right)
$$

for some integers $p^{\prime}, q^{\prime}, r^{\prime}>1, \operatorname{gcd}$ of $r^{\prime}$ and $q^{\prime}$ is strictly greater than 1 and $\left(r^{\prime \prime}, s^{\prime \prime}\right)$ is relatively prime.

We now prove that $B$ also is equivalent to the same desired form in (8.2). We are going to establish the first case, the second case is similar. Recall that $Q=\left(\begin{array}{cc}1 & 0 \\ -1 & 1\end{array}\right)$. If $Q^{T} B \tilde{U}=$ $\left(\begin{array}{ll}\frac{1}{q} & 0 \\ r & q\end{array}\right)$ for some unimodular integer matrix $\tilde{U}$, then $B \tilde{U}=\left(\begin{array}{cc}\frac{1+r q}{q} & q \\ r & q\end{array}\right)$. Note that $1+r q$ and $q^{2}$ is relatively prime. Thus there exist co-prime integers $u, v$ such that $(1+r q) u+q^{2} v=1$. Define $U=\left(\begin{array}{cc}u & -q^{2} \\ v & 1+r q\end{array}\right)$. Then $\operatorname{det}(U)=1$ and the lattice $B \tilde{U}\left(\mathbb{Z}^{2}\right)=B \tilde{U} U\left(\mathbb{Z}^{2}\right)$. Note that

$$
B \tilde{U} U=\left(\begin{array}{cc}
\frac{1+r q}{q} & q \\
r & q
\end{array}\right)\left(\begin{array}{cc}
u & -q^{2} \\
v & 1+r q
\end{array}\right)=\left(\begin{array}{cc}
\frac{1}{q} & 0 \\
r u+v q & q
\end{array}\right) .
$$

This shows that $B$ is equivalent to the desired form in (8.2). For the rest, we prove that the neither of these forms can form an Gabor orthonormal basis. By the Lemma 8.1 and Theorem 1.3 , we just need to prove that the octagon $\mathcal{O}_{8}$ is not a multi-tile for the matrices $\left(\begin{array}{cc}p & 0 \\ 0 & 1 / p\end{array}\right)$ and $\left(\begin{array}{cc}1 / p & 0 \\ 0 & p\end{array}\right)$ where $p>1$ is an integer. By the symmetry of the octagon, we just need to prove that $\mathcal{O}_{8}$ is not a multi-tile for the first one. Let $B_{p}:=\left(\begin{array}{cc}p & 0 \\ 0 & 1 / p\end{array}\right)$. To prove this, we first note that an elementary calculation shows that the area of $\mathcal{O}_{8}$ is 14 . If it multi-tiles by $B_{p}\left(\mathbb{Z}^{2}\right)$, then for almost every $x \in \mathbb{R}^{2}$, the cardinality of the set $\left(x+B_{p}\left(\mathbb{Z}^{2}\right)\right) \cap \mathcal{O}_{8}$ is $l=14$. To obtain a contradiction consider the rectangle $R_{p}:=[0,1) \times[0,1 / p)$ for $p>1$. It is a simple observation that $R_{p}$ can be covered $12 p$ by translations of $\mathcal{O}_{8}$ by the matrix $B_{p}$. This is a contradiction to the level of multi-tiling $l=14$, thus we have completed the proof. 


\section{Appendix A. Exponential Completeness}

In this appendix, we will study some special cases for exponential completeness and one of the cases will be used to prove Example 7.3. We will focus our attention on $\Lambda$ to be a subgroup of integers. The following proposition provides a full characterization of exponential completeness for $[0,1] \subset \mathbb{R}$ for integer lattices.

Proposition A.1. Let $\Lambda=p \mathbb{Z}, p \neq 0$. Then $\Lambda$ exponentially incomplete for $L^{2}[0,1]$ if and only if $p$ is an integer and $|p|>1$.

Proof. First assume that $p$ is an integer and $|p|>1$. Then in Definition 2.6 we take $\xi=1$ and we will have $\widehat{\chi_{[0,1]}}(1+p n)=0$ for all $n \in \mathbb{Z}$. This shows that such $\Lambda$ is exponentially incomplete.

Conversely, if $\Lambda$ is exponentially incomplete, then we can find $a \in \mathbb{R}$ such that $\widehat{\chi_{[0,1]}}(a+p n)=0$ for all $n \in \mathbb{Z}$. This means that $a+p n \in \mathbb{Z} \backslash\{0\}$ for all $n \in \mathbb{Z}$. Putting $n=0$ implies that $a \in \mathbb{Z} \backslash\{0\}$ and putting $n=1$ implies that $p$ is an integer and $p \neq-a$. Now let $n=a$ and $n=-a$, respectively. Then $a+a p$ and $a-a p$ are in $\mathbb{Z} \backslash\{0\}$ which imply that $|p| \neq 1$. Thus, $|p|>1$ and this completes the proof.

The classification of exponential incomplete lattices in higher dimensions is not straight forward as in the case dimension one. Given a vector $\mathbf{v}=\left(v_{1}, \ldots, v_{d}\right) \in \mathbb{R}^{d}$, we define the $\mathbb{R}$-subgroup $G(\mathbf{v})$ generated by the entries of $\mathbf{v}$ as

$$
G(\mathbf{v}):=\left\{v_{1} n_{1}+\ldots+v_{d} n_{d}:\left(n_{1}, \ldots, n_{d}\right) \in \mathbb{Z}^{d}\right\}=\left\{\langle\mathbf{v}, n\rangle: n \in \mathbb{Z}^{d}\right\} .
$$

Proposition A.2. Let $B=\left(\begin{array}{ccc}- & \mathbf{v}_{1} & - \\ - & \vdots & - \\ - & \mathbf{v}_{d} & -\end{array}\right), d>1$. Suppose that there exists $\mathbf{v}_{k}$ such that $G\left(\mathbf{v}_{k}\right)=p \mathbb{Z}$ for some integer $p$. If $|p|>1$, then $B\left(\mathbb{Z}^{d}\right)$ is exponentially incomplete in $L^{2}\left([0,1]^{d}\right)$.

Proof. We note that for any $\alpha=\left(\alpha_{1}, \ldots, \alpha_{d}\right) \in \mathbb{R}^{d}$ and any $n \in \mathbb{Z}^{d}$,

$$
\widehat{\chi_{[0,1]^{d}}}(\alpha+B n)=\prod_{i=1}^{d} \widehat{\chi_{[0,1]}}\left(\alpha_{i}+\left\langle\mathbf{v}_{i}, n\right\rangle\right)
$$

Clearly, if one of $G\left(\mathbf{v}_{k}\right)=p \mathbb{Z}$ for some $p>1$, then we just let $\alpha_{k}=1$ and obtain that $\widehat{\chi_{[0,1]}}\left(1+\left\langle\mathbf{v}_{k}, n\right\rangle\right)=0$ for all $n \in \mathbb{Z}^{d}$. This completes the proof.

Remark: If $B$ is an integral matrix, then one can reduce $\mathrm{B}$ to a lower triangular matrix with $b_{11}=p$. This means that $G\left(v_{1}\right)=p \mathbb{Z}$, hence the result holds if $|p|>1$. However, the assumption $|p|>1$ is not necessary, thus the converse of the proposition does not hold in general. For this, take $B=\left(\begin{array}{ll}p & 0 \\ r & q\end{array}\right)$ where $p$ is a non-zero integer, $q$ is an integer with $|q|>1,(p, q)=1$ and $r$ is a real number. Take $\alpha=(p, p+r)$. Then for $m=-1$, we have $\widehat{\chi_{[0,1]}}(p+r(1+m)+q n)=0$ since $p$ and $q$ are co-primes. For $m \neq-1$, we have $\widehat{\chi_{[0,1]}}(p(1+m))=0$. Thus, for all $\mathbf{n}=(m, n) \in \mathbb{Z}^{d}$ we have $\widehat{\chi_{[0,1]}}(\alpha+B \mathbf{n})=0$, and the exponential incompleteness holds. 
The following theorem is a special case in dimension $d=2$ which fits into our needs for the proof of Example 7.3.

Theorem A.3. Let $B$ be a $2 \times 2$ rational matrix with $\operatorname{det}(B)=1$. Then $B\left(\mathbb{Z}^{2}\right)$ is exponentially incomplete for $L^{2}[0,1]^{2}$ if and only if $B$ is equivalent to $\left(\begin{array}{cc}\frac{1}{q^{\prime}} & 0 \\ r^{\prime} & q^{\prime}\end{array}\right)$ or $\left(\begin{array}{cc}p^{\prime} & 0 \\ \frac{r^{\prime \prime}}{s^{\prime \prime}} & \frac{1}{p^{\prime}}\end{array}\right)$ for some integers $p^{\prime}, q^{\prime}, r^{\prime}>1$ with the greatest common divisor $\operatorname{gcd}\left(r^{\prime}, q^{\prime}\right)>1$ and $r^{\prime \prime}$ and $s^{\prime \prime}$ are relatively prime.

Proof. The sufficiency follows from Proposition A.2 since $G\left(r^{\prime}, q^{\prime}\right)=d \mathbb{Z}$ for $d:=\operatorname{gcd}\left(r^{\prime}, q^{\prime}\right)$ and $G\left(p^{\prime}, 0\right)=p^{\prime} \mathbb{Z}$. We now prove the necessity. By Corollary 2.5, there exists an integer matrix $U$ of determinant one such that $B U$ is lower triangular. Since $\operatorname{det}(B)=1$ and the matrix is rational, we can assume that

$$
B=\left(\begin{array}{cc}
\frac{p}{q} & 0 \\
\frac{r}{s} & \frac{q}{p}
\end{array}\right)
$$

and the pairs of integers $(p, q)$ and $(r, s)$ are respectively relatively prime. Writing $\mathbf{x}_{0}=\left(x_{1}, x_{2}\right)$, $B\left(\mathbb{Z}^{2}\right)$ is exponentially incomplete only if we have some $\mathbf{x}_{0}$ such that

$$
\widehat{\chi_{[0,1]}}\left(x_{1}+\frac{p}{q} n_{1}\right) \widehat{\chi_{[0,1]}}\left(x_{2}+\frac{r}{s} n_{1}+\frac{q}{p} n_{2}\right)=0
$$

holds for all integers $\left(n_{1}, n_{2}\right)$. Note that the zeros of $\widehat{\chi_{[0,1]}}$ are $\mathbb{Z} \backslash\{0\}$. Putting $n_{1}=1$ and $n_{2}=0$ yields

$$
\widehat{\chi_{[0,1]}}\left(x_{1}+\frac{p}{q}\right)=0 \text { or } \widehat{\chi_{[0,1]}}\left(x_{2}+\frac{r}{s}\right)=0 .
$$

Thus $x_{1}=k_{1}-p / q$ or $x_{2}=k_{2}-r / s$ for some $k_{1}, k_{2} \in \mathbb{Z} \backslash\{0\}$.

Case (1) $x_{1}=k_{1}-p / q$. We then put $n_{1}=2$ and $n_{2}=0$ to conclude that $x_{1}+2 p / q \in \mathbb{Z} \backslash\{0\}$ or $x_{2}+2 r / s \in \mathbb{Z} \backslash\{0\}$.

Sub-case (i) $x_{1}+2 p / q \in \mathbb{Z} \backslash\{0\}$. In this subcase, we have $k_{1}+p / q \in \mathbb{Z} \backslash\{0\}$, which implies $p / q$ is an integer. Thus, $q=1$. We now argue that $p>1$ and hence it must be the second form. Indeed, we know that $x_{1} \in \mathbb{Z}$. If it happens that $p=1$, we can take $n_{1}=-x_{1}$, we will have $x_{2}-x_{1} r / s+n_{2} \in \mathbb{Z} \backslash\{0\}$ for any integer $n_{2}$. Put $n_{2}=0$. We obtain that $x_{2}=\ell+x_{1} r / s$ for some integer $\ell$. However, we then take $n_{2}=-\ell$, we will have $0 \in \mathbb{Z} \backslash\{0\}$, which is a contradiction. Thus, $p>1$ and we proved this subcase.

Sub-case (ii) $x_{2}+2 r / s \in \mathbb{Z} \backslash\{0\}$. In this subcase, $x_{2}=\ell-2 r / s$ for some non-zero integer $\ell$. As subcase (i) does not hold, considering $n_{1}=2$ and any integers $n_{2}$ we have $\ell+n_{2} \frac{q}{p} \in \mathbb{Z} \backslash\{0\}$ for all $n_{2}$. Putting $n_{2}=1$, we conclude that $q / p$ is an integer and thus $p=1$. Note that $q>1$ also. Otherwise, we can put $n_{2}=-\ell$ and we obtain a contradiction. Finally, we have for any integer $n_{1}, n_{2}$

$$
\widehat{\chi_{[0,1]}}\left(k_{1}+\frac{n_{1}-1}{q}\right)=0 \text { or } \widehat{\chi_{[0,1]}}\left(\ell+\frac{\left(n_{1}-2\right) r}{s}+n_{2} q\right)=0
$$

Claim 1: $s=1$

Suppose that $s>1$. If $q=2$, we consider an even number $n_{1}$, then $k_{1}+\left(n_{1}-1\right) / 2$ cannot be an integer, so $\ell+\left(n_{1}-2\right) r / s+2 n_{2}$ are all non-zero integers. Thus, putting $n_{1}=4,2 r / s$ is an 
integer, which means $s=2$ is even. we must have $\ell+\left(n_{1} / 2-1\right) r+2 n_{2}$ are non-zero integers. In this case, $r$ must be even, otherwise, 2 and $r$ is relatively prime and we can find $N, n_{2}$ (and $\left.n_{1}=2(N+1)\right)$ such that $N r+2 n_{2}=-\ell$, we obtain $\ell+\left(n_{1} / 2-1\right) r+2 n_{2}=0$, which is a contradiction. Hence, $r, s$ is not relatively prime. This is a contradiction again. Thus, $s=1$. If $q>2$, then nwe put $n_{1}=3$, we will have $k_{1}+2 / q$ or $\ell+r / s+q n_{2}$ is an non-zero integer. Clearly, the first one cannot be as $q>2$, We must have $\ell+r / s+q n_{2}$ is an integer, which forces $r / s$ is an integer and $s=1$ follows.

Claim 2: gcd of $r, q$ must be strictly greater than 1.

Now, $x_{1}, x_{2}$ are integers and we have $x_{1}+n_{1} / q$ or $x_{2}+n_{1} r+q n_{2}$ is a non-zero integer for any integers $n_{1}, n_{2}$. Suppose that the gcd of $r, q$ is one. Then we can find $n_{1}, n_{2}$ such that $n_{1} r+q n_{2}=-x_{2}$. If $q$ does not divide $x_{2}$, then $q$ does not divide $n_{1}$. We will have both $x_{1}+n_{1} / q$ and $x_{2}+n_{1} r+q n_{2}$ are not non-zero integers, which is a contradiction. If $q$ divides $x_{2}$, then we must have $x_{2}=N q$. Take $n_{1}=-x_{1} q$ and $n_{2}=x_{1} r-N$, then we have all $x_{1}+n_{1} / q$ or $x_{2}+n_{1} r+q n_{2}$ equal zero. A contradiction again. Therefore, gcd of $r, q$ must be strictly greater than one.

Case (2) $x_{2}=k_{2}-r / s$ we then take $n_{1}=1$ and $n_{2}=1$ to conclude that $x_{1}+p / q$ or $k_{2}+q / p$ must be a non-zero integer. The first case is back to Case(1), so we are done. In the second case, $p=1$ and for any integer $n_{1}, n_{2}, x_{1}+n_{1} / q$ or $k_{2}+\left(n_{1}-1\right) r / s+n_{2} q$ is a non-zero integer.

If we take $n_{1}=0$, then we conclude that $x_{1}$ or $k_{2}-r / s+n_{2} q$ are non-zero integers. If $x_{1}$ is an integer, then we have the same set of equations as in (A.2). We can then argue similarly as in Claim 1 and 2 to settle this case. Finally, if $k_{2}-r / s+n_{2} q$ is a non-zero integer, we have $s=1$ and it remains to claim that gcd of $r, q$ is strictly greater than one. Indeed, $x_{2}$ is an integer. If gcd of $r, q$ is one and $q$ does not divide $x_{2}$, we can find $N_{1}, N_{2}$ such that $-x_{2}=N_{1} r+N_{2} q$ is a non-zero integer. In this case, $x_{1}+N_{1} / q$ is a non-zero integer. concluding that $x_{1}=k-N_{1} / q$ for some non-zero integer $k$. We now have $k+\left(n_{1}-N_{1}\right) / q$ or $x_{2}+n_{1} r+n_{2} q$ is a non-zero integer. However, we can $n_{1}=N_{1}-k q$, and $n_{2}=k r+N_{2}$. Then $x_{2}+n_{1} r+n_{2} q=0$ and $k+\left(n_{1}-N_{1}\right) / q=0$ also, which is a contradiction. Thus, gcd of $r, q$ is strictly greater than one.

\section{REFERENCES}

[1] D. Barbierie, E. Hernandez, A. Mayeli, Tiling by lattices for locally compact abelian groups, Comptes rendus Mathematique, Vol. 355, Issue 2, Feb. 2017, pages 193-199. [2]

[2] L. De Carli, A. Mizrahi and A. Tepper, Three problems on exponential bases, https://arxiv.org/pdf/1710.05342.pdf, 2017. [18]

[3] Ole Christensen, An Introduction to Frames and Riesz Bases, Birkhaüser, 2002. [1]

[4] R. Chung and C.K. Lai, Non-symmetric convex polytope and Gabor orthnonormal bases, To appear in Proc. Amer. Math. Soc. [19]

[5] D. Dutkay and C.K. Lai, Uniformity of measures with Fourier frames, Advances in Mathematics 252 (2014), 684-707. [3, 19]

[6] B. Farkas, M. Matolsci, Peter Mora, On Fuglede's Conjecture and the Existence of Universal Spectra, Journal of Fourier Analysis and Applications, 12 (2006), p 483-494. [2]

[7] J. Feldman and F. P. Greenleaf, Existence of Borel transversals in groups, Pacific J. Math. 25 (1968), 455-461. [5]

[8] G. Folland, Harmonic Anlaysis in phases spaces, Princeton University Press., 1989. [11]

[9] B. Fuglede, Commuting self-adjoint partial differential operators and a group theoretic problem, J. Funct. Anal. 16 (1974), 101-121. [2] 
[10] J.-P Gabardo and Y.-Z Li, Density results for Gabor systems associated with periodic subsets of the real line, J. Approx. Theory, 157 (2009), 172-192. [10]

[11] D. Gabor, Theory of communication, J. Inst. Elec. Engrg. (London) 93 (1946) 429-457. [1]

[12] M. de Gosson, Symplectic Methods in Harmonic Analysis and in Mathematical Physics, Birkhuser, 2011. $[11]$

[13] K. H. Gröchenig, Foundations of Time-Frequency Analysis, Applied and Numerical Harmonic Analysis, Birkhuser, 2001. [1, 6, 11]

[14] K. H. Gröchenig, The mystery of Gabor frames, 20 (2014), 865-895. [3, 7]

[15] D. Han and Y. Wang, Lattice Tiling and The Weyl-Heisenberg Frames, Geo. Funct. Anal., 11 (2001), 742-758. [1, 18, 19]

[16] D. Han and Y. Wang, The existence of Gabor Bases, Contemporary Math. 345 (2004), 183-192. [2, 4, 6, $13,18]$

[17] A. Iosevich, N. H. Katz and T. Tao Convex bodies with a point of curvature do not have Fourier bases, Amer. J. Math., 123 (2001), 115-120. [19]

[18] A. Iosevich, A. Mayeli, Gabor orthogonal bases and convexity, preprint. [19]

[19] A. Iosevich, A. Mayeli and J. Pakianathan, The Fuglede Conjecture holds in $\mathbb{Z}_{p} \times \mathbb{Z}_{p}$, Anal. PDE 10 (2017), no. 4. [2]

[20] P. Jorgensen, S. Pedersen, Spectral theory for Borel sets in $\mathbb{R}^{n}$ of finite measure, J. Funct. Anal. 107 (1992) 72-104. [7]

[21] M. Kolountzakis, Multiple lattice tiles and Riesz bases exponentials, Proc. Amer. Math. Soc,143 (2015), 741-747. [2]

[22] M. Kolountzakis and M. Matolcsi, Tiles with no spectra, Forum Math., 18, (2006), no. 3, 519-528. [2, 19]

[23] M. Kolountzakis, Non-symmetric convex domains have no basis of exponentials, Illinois journal of mathematics 44(3), March 1999 [19]

[24] J.-P Gabardo, C.-K Lai and Y. Wang, Gabor orthonormal bases generated by the unit cube, J. Funct. Anal., 269 (2015), 1515-1538. [6]

[25] M. Matolcsi, Fugledes conjecture fails in dimension 4, Proc. Amer. Math. Soc., 133 (2005), 3021-3026. [19]

[26] J. Ramanathan and T. Steger, Incompleteness of sparse coherent states, Appl. Comp. Harm. Anal. 2 (1995), 148-153. [1]

[27] A. Ron and Z. Shen, Weyl-Heisenberg frames and Riesz bases in $L_{2}\left(\mathbb{R}^{d}\right)$, Duke Math. J., 89 (1997), $237-282$. $[7]$

[28] T. Tao, Fuglede's conjecture is false in 5 and higher dimensions, Math. Res. Lett. 11 (2004), no. 2-3, 251-258. [2, 19]

[29] J. Lagarias and Y. Wang, Spectral Sets and Factorizations of Finite Abelian Groups, J. Funct. Anal., 145 (1997), 73-98. [7]

[30] Youming Liu, Yang Wang, The uniformity of non-uniform Gabor bases, Adv. Comput. Math., 18 (2003), 345-355. [2]

Chun-Kit lai, Department of Mathematics, College of Science and Engineering, San Francisco State Univesrity, San Francisco, USA

E-mail address: cklai@sfsu.edu

Azita Mayeli, Department of Mathematics and Computer Science, Queensborough and The Graduate Center, City University of New York, USA

E-mail address: AMayeli@gc.cuny.edu 